\title{
Arquiteturas de Referência para Sistemas-de-Sistemas: um Mapeamento Sistemático
}

\section{Title: Reference Architectures for Systems-of-Systems: a Systematic Mapping}

Priscilla Elizabeth Pereira Batista ${ }^{1,2}$, Renato de Freitas Bulcão Neto ${ }^{2}$, Carlos

Eduardo de Barros Paes³, Eliomar Araújo de Lima², Adair José Rohling Valdemar Vicente Graciano Neto ${ }^{2}$

${ }^{1}$ Tribunal de Justiça do Estado de Goiás (TJGO)

Goiânia, Goiás - Brasil

${ }^{2}$ Instituto de Informática - Universidade Federal de Goiás (UFG)

Goiânia, Goiás - Brasil

${ }^{3}$ Pontifícia Universidade Católica de São Paulo (PUC-SP)

São Paulo, São Paulo - Brasil

${ }^{4}$ Universidade Tecnológica Federal do Paraná (UTFPR)

Guarapuava, Paraná - Brasil

pepbatista@tjgo.jus.br, \{bulcao, eliomar.lima\}@ufg.br, carlosp@pucsp.br, adairrohling@utfpr.edu.br, valdemarneto@ufg.br

\begin{abstract}
Systems-of-Systems (SoS) are a class of complex systems that often involve Information Systems as their constituents. However, when systems are required to become constituents of a SoS, they should meet specific requirements. In this sense, a reference architecture $(R A)$ can bring together the requirements and design details necessary to enable a system to become a SoS constituent, in addition to assuring SoS quality and potential for reusing the designed architectures. An investigation of the state of the art in the field can, therefore, help to understand how SoS has in fact benefited from RAs and what still needs to be progressed. The main contribution of this article is to present the results of a systematic mapping on the RAs proposed in SoS context. 23 studies out of 361 studies retrieved from 5 bases were included for analysis and extraction. Results reveal, among other information, the domains in which RAs are being applied in SoS, such as smart grids, as well as how they have been developed, which involve the use of reference models, architecture frameworks and design processes.
\end{abstract}

Keywords. Systems-of-Systems; Reference Architecture; Systematic Mapping.

Cite as: $\quad$ Batista, P. E. P., Bulcão Neto, R. F., Paes, C. E. B., Lima, E. A., Rohling, A. J. \& Graciano Neto, V. (2021). Reference Architectures for Systems-of-Systems: a Systematic Mapping (Arquiteturas de Referência para Sistemas-de-Sistemas: um Mapeamento Sistemático). iSys: Revista Brasileira de Sistemas de Informação (Brazilian Journal of Information Systems), 14(1), 119-153. DOI: 10.5753/isys.2021.988 
Resumo. Sistemas-de-Sistemas (SoS) são uma classe de sistemas complexos que frequentemente envolvem Sistemas de Informação como seus constituintes. Entretanto, para que sistemas possam tornar-se constituintes de um SoS, é importante que atendam a requisitos específicos, tais como interoperabilidade $e$ independência operacional e gerencial dos constituintes. Neste sentido, uma arquitetura de referência $(A R)$ pode reunir os requisitos e detalhes de design necessários para habilitar um sistema a tornar-se constituinte de um SoS, além de reforçar sua qualidade e potencial de reúso das arquiteturas concebidas. Uma investigação sobre o estado da arte na área pode, portanto, auxiliar a compreender como SoS tem de fato se beneficiado das ARs e o que ainda é necessário progredir. A principal contribuição deste artigo é apresentar resultados de um mapeamento sistemático sobre as ARs que têm propostas no contexto de SoS, bem como os modelos, técnicas e processos utilizados para sua concepção. De um total de 361 estudos recuperados de 5 bases, 23 estudos foram incluídos para análise e extração. Resultados revelam, dentre outras informações, os domínios em que as arquiteturas de referência estão sendo aplicadas em SoS, tais como smart grids, bem como a forma que elas têm sido desenvolvidas, que envolvem o uso de modelos de referência, frameworks de arquitetura e processos de concepção.

Palavras-chave. Sistemas de Sistemas; Arquitetura de Referência; Mapeamento Sistemático

\section{Introdução}

Sistemas-de-Sistemas (SoS, do inglês Systems-of-Systems) são conjuntos de sistemas independentes que interoperam para atingir objetivos comuns. SoS valem-se das funcionalidades individuais oferecidas por seus sistemas constituintes para, juntos, entregarem comportamentos mais complexos e atingirem resultados que os sistemas constituintes não seriam capazes de entregar individualmente. Acompanhando tendências mundiais, organizações têm sido motivadas a interoperar seus sistemas de informação (SI) com outros sistemas externos de modo a estabelecer alianças de negócio, gerando tipos específicos de SoS chamados de Sistemas-de-Sistemas de Informação (SoIS, do inglês Systems-of-Information Systems). Nesta modalidade de SoS, SI são impelidos a interoperar com outros SI de outras organizações e também com outros tipos de sistemas como drones e sistemas de tráfego inteligente para criarem ecossistemas de valor, ainda que mantenham sua independência operacional e gerencial. Exemplos desta tendência são as cidades inteligentes, que envolvem inúmeros SI para gerirem a cidade enquanto interoperam com sistemas em diversos níveis e de diversos tipos [Graciano Neto et al. 2017a].

Para alinharem seus SI aos requisitos de independência operacional e gerencial e os habilitarem a serem constituintes de um SoS (ou SoIS), é essencial que seus SI evoluam de modo a serem capazes de interoperar com outros sistemas de interesse. No entanto, a interoperabilidade é justamente um dos maiores desafios enfrentados pelos SI modernos [Maciel et al. 2017] e a independência operacional de constituintes tem sido, por si só, um tema recente de pesquisa [Teixeira et al. 2019]. Nesse sentido, entende-se que uma arquitetura de referência (AR) pode auxiliar na concepção de SoS e de suas partes. ARs referem-se a um tipo especial de arquitetura de software que captura a essência das 
arquiteturas de um conjunto de sistemas de software de um determinado domínio [Nakagawa et al. 2012]. As ARs são arquétipos, isto é, arquiteturas abstratas que encapsulam a estrutura e funcionalidades que deveriam ser oferecidas por um sistema para auxiliar na uniformização das características dos sistemas aderentes a elas. No caso do SoS, uma AR deveria, por exemplo, incluir parâmetros referentes a interoperabilidade e independências gerencial e operacional dos constituintes. Assim, ARs propostas para o SoS podem guiar arquitetos a respeito de como desenvolver constituintes, como estabelecer enlaces entre eles para formar um SoS, ou mesmo utilizar as ARs para guiar a evolução de SI existentes para serem capazes de engajar-se de modo bem sucedido em um SoS.

Neste sentido, o intuito deste artigo é trazer resultados de um mapeamento sistemático para (i) compreender se e como as ARs têm sido propostas no contexto de SoS e (ii) obter evidência sobre os processos, métodos e técnicas adotados para tal, além (iii) dos domínios nos quais tais ARs têm sido propostas no contexto de SoS e SoIS Reitera-se que, uma vez que todo SoIS é um SoS (embora a recíproca não seja verdadeira) [Graciano Neto et al. 2021], ao conduzir um mapeamento sistemático com foco nos SoS, os SoIS seriam cobertos de modo colateral. Entretanto, para assegurar que os SoIS sejam explicitamente cobertos, os termos e seus sinônimos foram utilizados na busca e na análise dos resultados com menção explícita aos SoIS para compreender se eles têm sido tratados especificamente nas pesquisas. Tal decisão foi tomada para (i) contribuir com um dos Grandes Desafios em Sistemas de Informação para a Década de 2016-2026 [Boscarioli et al. 2017], investigando como ARs têm sido tratadas no campo de SoS, e averiguar se o campo específico de SoIS também tem sido coberto, (ii) identificar oportunidades de pesquisa que envolvam o campo de SoIS e (iii) enfatizar a intenção de levantar os requisitos necessários para a elaboração de uma AR para guiar a concepção e evolução de SI de modo a poderem tornar-se constituintes de um SoS (que é considerado um SoIS se houver a presença de SI). Como os SoIS são sistemas ainda escassos e pouco estudados, preferiu-se incluir o SoS no mapeamento para possibilitar uma amostra mais ampla de estudos. Assim, SoIS serão mencionados explicitamente ao longo do texto para tratar as especificidades de SoIS; nos momentos restantes, entende-se que tratar de SoS engloba SoIS.

As contribuições esperadas para o mapeamento são, portanto: (i) um levantamento do estado da arte sobre proposição e uso de AR no contexto de SoS (e SoIS), (ii) identificação do nível (SoS, constituinte ou componentes de constituintes ou quaisquer outros elementos de software) em que AR têm sido propostas no contexto de SoS, (iii) levantamento de requisitos para concepção de AR no contexto de SoS, e (iv) identificação de oportunidades de pesquisa relativas a AR para SoS/SoIS. Foi utilizado um protocolo de pesquisa para o mapeamento sistemático que consiste na busca sistemática em bases de publicações científicas selecionadas. Finalmente, os estudos identificados foram selecionados e analisados para extrair as informações necessárias e responder às questões de pesquisa levantadas [Felizardo et al. 2017]. Os resultados do mapeamento mostram que a área de ARs para SoS ainda é pouco explorada. Os estudos analisados focam, principalmente, em ARs para o SoS como um todo em detrimento de seus constituintes, mediadores ou links de interoperabilidade. Também foi possível verificar que ainda há muitas oportunidades de pesquisa com a criação de ARs nos mais diversos domínios, posto que somente 10 domínios foram mapeados, dos quais 2 domínios possuem $43 \%$ dos 
estudos (smart grid e segurança).

O restante do artigo está organizado da seguinte forma. A Seção 2 apresenta uma breve fundamentação teórica, bem como os trabalhos correlatos. A Seção 3 apresenta o protocolo do mapeamento sistemático bem como sua condução e resultados. A Seção 4 lista as oportunidades de pesquisa identificadas juntamente com uma discussão dos achados. A Seção 5 explicita as ameaças à validade da pesquisa. Finalmente, a Seção 6 apresenta as considerações finais e os trabalhos futuros.

\section{Fundamentação Teórica e Trabalhos Correlatos}

O SoS é distinguido por duas dimensões bem definidas, assim postuladas por Maier [Maier, 1998]: (i) independência operacional dos constituintes, uma vez que preservam sua independência e operação própria ainda que trabalhem dentro do escopo do SoS; e (ii) independência gerencial dos constituintes, uma vez que organizações e entidades diferentes são proprietários dos constituintes e consequentemente gerenciam e tomam decisões sobre os sistemas que fazem parte de um SoS. Partindo destas dimensões, Maier [Maier, 1998] propõe as seguintes características adicionais para SoS: (i) o desenvolvimento evolucionário do SoS, isto é, à medida que os constituintes e os propósitos do SoS mudam, o SoS também evolui como consequência; (ii) distribuição, uma vez que o funcionamento do SoS depende de tecnologias de comunicação que habilitem a devida comunicação entre seus constituintes; e (iii) comportamento emergente, ou seja, um comportamento global do SoS projetado a partir de interações entre os constituintes, e que corresponde ao resultado combinado das funcionalidades de seus constituintes.

Essas características também se aplicam aos SoIS. SoIS são SoS que possuem um ou mais Sistemas de Informação - SIs entre seus constituintes, podendo ou não haver outros tipos de sistemas incluídos [Graciano Neto et al. 2017b]. Geralmente, sistemas de informação que constituem o SoIS são sistemas que fazem uso intensivo de software (do inglês, software-intensive). Devido à variedade de sistemas envolvidos, a interoperabilidade se torna um fator preponderante para o funcionamento desses sistemas. Os SoIS estão representados em diversos domínios, como as cidades inteligentes [Mendes et al. 2018].

Quando não existem padrões definidos para o desenvolvimento de um sistema de interesse, a interoperabilidade é um dos fatores que pode ser prejudicado, tornando difícil fazer com que sistemas individuais que não foram desenvolvidos de forma padronizada trabalhem cooperativamente. Nesse contexto, a definição de uma AR para SoS torna-se importante, pois permite padronizar o processo de construção da arquitetura tanto de um SoS como de um SoIS bem como de seus elementos internos (constituintes, mediadores e demais elementos de interoperabilidade). AR é um tipo de arquitetura que permite a padronização no desenvolvimento de software, facilitando o reaproveitamento de recursos e soluções e possibilitando maior interoperabilidade entre os SIs [Nakagawa et al. 2017]. ARs já existem em vários domínios, tais como o domínio espacial [Rohling et al., 2019] e atributos de qualidade específicos, como a Sustentabilidade, têm sido tratados neste contexto [Volpato et al., 2017]. Nesse sentido, ARs podem ser úteis para garantir a interoperabilidade dos SIs que irão compor um SoS. 
Trabalhos correlatos. Durante a pesquisa, não foram identificados estudos sistemáticos que relacionam Arquitetura de Referência e SoS. Esta falta de estudos deve-se, em parte, ao fato de tratar-se de uma área de pesquisa recente, principalmente no que tange à proposição de arquiteturas de referências para SoS. Além disso, de acordo com Cadavid et al. (2020), os estudos secundários na área de SoS estão ainda imaturos e, ainda que o número de estudos desta natureza esteja aumentando, eles ainda encontram-se desconexos entre si.

Algumas revisões sistemáticas que tratam de arquiteturas de SoS já foram publicadas, sendo Cadavid et al. (2020) um exemplo. O trabalho apresenta um estudo terciário com intuito de expor o estado da arte nas arquiteturas de SoS. Nos 19 estudos analisados, foi possível perceber que as pesquisas relacionadas a SoS têm atingido avanços significativos e existe uma diversidade de tópicos abordados, tais como descrição arquitetural de SoS, uso de abordagens dirigidas por modelos para SoS, bem como arquiteturas de SoS em domínios específicos, que incluem cidades inteligentes, IoT e Ecossistemas de Software. Entretanto, o estudo também relata uma desconexão nos tópicos investigados pela comunidade de pesquisa, com lacunas de pesquisa ainda existentes em etapas específicas do processo de design arquitetural e como tratar atributos de qualidade específicos em SoS.

Guessi et al. (2015) apresenta uma revisão sistemática sobre as descrições de arquiteturas de software para SoS. Apesar de haver vários estudos nessa área, ainda havia falta de consenso sobre as linguagens usadas para descrição arquitetural de SoS e qual o nível de formalismo necessário nas linguagens para que pudessem ser úteis na avaliação e evolução do SoS. O trabalho aborda apenas arquiteturas concretas (não abstratas, como ARs) e lidava particularmente com as notações utilizadas até então para realizar descrição arquitetural, tais como UML e SysML.

Gatica et al. (2018) realizou um mapeamento sistemático que cobriu 22 estudos e identificou propostas de avaliação arquitetural com foco especial em atributos de qualidade para SoS. Em Lana et al. (2019) foi conduzido um mapeamento sistemático que recuperou 26 estudos com o estado da arte nas técnicas e linguagens utilizadas para modelar os requisitos de um SoS

Valle et al. (2020) conduziu um mapeamento sistemático que visava avaliar as abordagens que foram propostas para descrever ARs. Foram incluídos 21 estudos neste mapeamento, sendo que a maioria apresentou baixo nível de maturidade. De acordo com os pesquisadores, interoperabilidade e dinamismo, que são características essenciais dos sistemas complexos, foram abordados de modo incipiente nas pesquisas.

Nos últimos anos, diversos estudos sobre arquitetura de software em SoS têm sido publicados. $\mathrm{O}$ foco nos estudos relacionados citados estão em arquitetura de software para SoS, e não arquitetura de referência, como é o caso do estudo aqui relatado. Nota-se, portanto, a falta de estudos sistemáticos que analisam as ARs no contexto de SoS. Este estudo consiste em um passo adiante nesse caminho. Na próxima seção, o protocolo do estudo é apresentado, bem como a condução e os resultados. 


\section{Mapeamento Sistemático}

Para conduzir o mapeamento sistemático, a primeira etapa consistiu na criação do protocolo. A Figura 1 apresenta o processo de mapeamento sistemático utilizado neste trabalho.

A primeira etapa do protocolo de pesquisa foi elaborar as questões de pesquisa condizentes com o objetivo do mapeamento sistemático. Em seguida, realizou-se a análise da área de pesquisa e a elaboração e calibração da string de busca. Com a string definida, foram escolhidas as bases de busca relevantes para realização da pesquisa. Os critérios de inclusão e exclusão dos estudos foram estabelecidos e um formulário de pesquisa foi criado para a extração das informações.
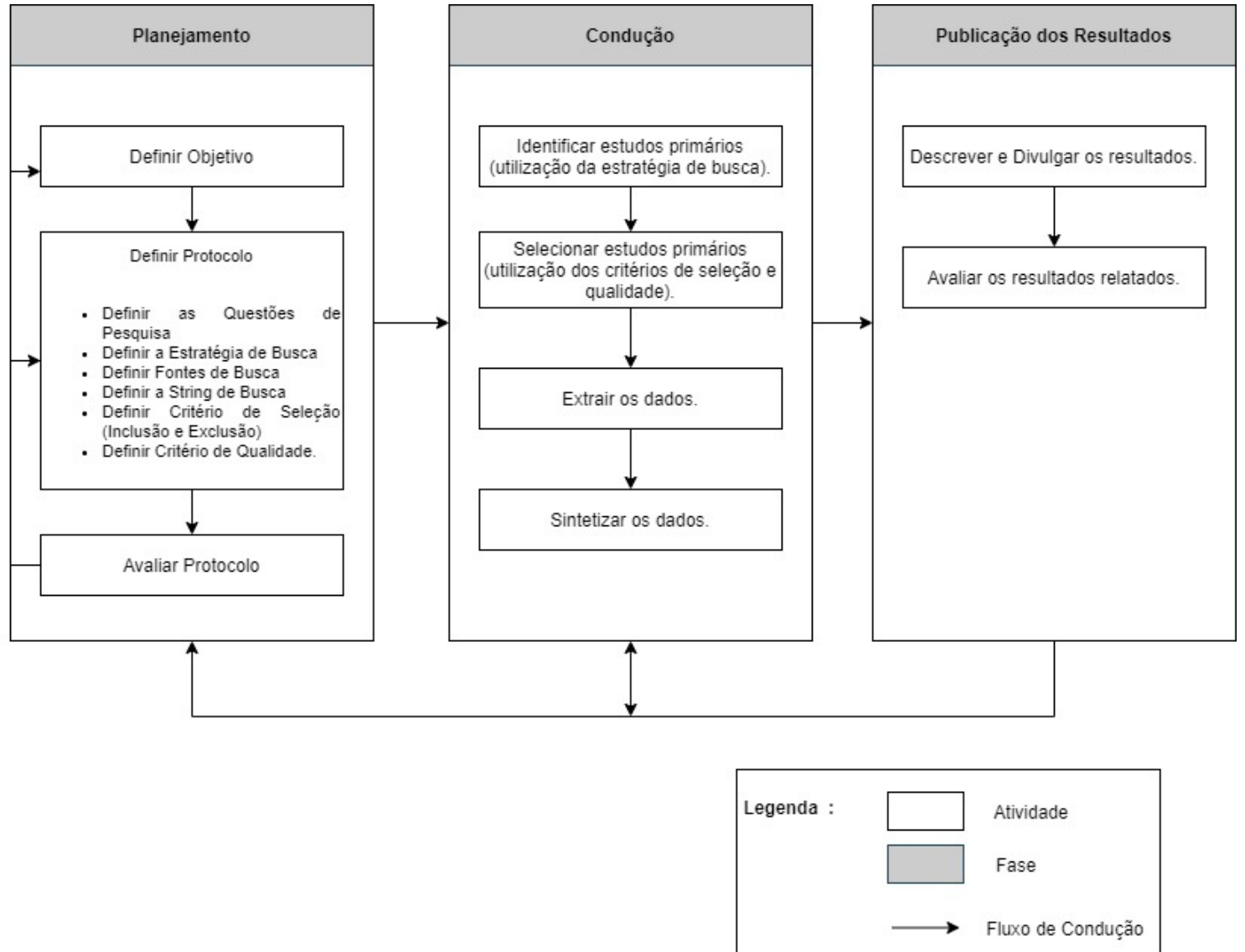

Figura 1. Etapas do Mapeamento Sistemático [Felizardo et al. 2017].

\subsection{Questões de Pesquisa}

A Tabela 1 apresenta as Questões de Pesquisa (QP) e suas respectivas justificativas.

Tabela 1. Questões de Pesquisa.

\begin{tabular}{|c|l|l|}
\hline & Questão de Pesquisa & \multicolumn{1}{|c|}{ Objetivo } \\
\hline QP 1. & $\begin{array}{l}\text { Quando e em que veículos } \\
\text { os estudos têm sido } \\
\text { publicados? }\end{array}$ & $\begin{array}{l}\text { Identificar onde o assunto é mais abordado e em que } \\
\text { anos as publicações se concentram. }\end{array}$ \\
\hline
\end{tabular}




\begin{tabular}{|c|c|c|}
\hline QP 2. & $\begin{array}{l}\text { Quais arquiteturas de } \\
\text { referência têm } \text { sido } \\
\text { propostas no contexto de } \\
\text { SoS/SolS? }\end{array}$ & Identificar as AR já existentes em SoS/SolS. \\
\hline $\begin{array}{l}\text { QP } \\
2.1 .\end{array}$ & $\begin{array}{l}\text { Quais modelos de referência } \\
\text { são usados para criar } \\
\text { arquitetura de referência? }\end{array}$ & $\begin{array}{l}\text { Identificar os métodos ou modelos de referência } \\
\text { utilizados para criar ARs em SoS. }\end{array}$ \\
\hline $\begin{array}{l}\text { QP } \\
2.2 .\end{array}$ & $\begin{array}{l}\text { Para quais domínios de } \\
\text { aplicação elas têm sido } \\
\text { propostas? }\end{array}$ & $\begin{array}{l}\text { Identificar quais domínios de aplicação dos SoS } \\
\text { possuem AR. }\end{array}$ \\
\hline $\begin{array}{l}\text { QP } \\
2.3 .\end{array}$ & $\begin{array}{l}\text { Quais estilos arquiteturais } \\
\text { têm sido utilizados nessas } \\
\text { AR? }\end{array}$ & Identificar os estilos arquiteturais mais comuns nas ARs. \\
\hline $\begin{array}{l}\text { QP } \\
2.4 .\end{array}$ & $\begin{array}{l}\text { Quais tipos de métodos são } \\
\text { usados para avaliar as AR? }\end{array}$ & Identificar como as AR têm sido avaliadas. \\
\hline QP 2.5 & $\begin{array}{l}\text { Quais as tecnologias } \\
\text { recomendadas/prescritas } \\
\text { nestas arquiteturas? }\end{array}$ & $\begin{array}{l}\text { Identificar quais tecnologias a AR indica para a } \\
\text { implementação do SoS. }\end{array}$ \\
\hline QP 2.6 & $\begin{array}{l}\text { Quais os atributos de } \\
\text { qualidade priorizados nas } \\
\text { arquiteturas? }\end{array}$ & $\begin{array}{l}\text { Identificar quais atributos de qualidade de arquitetura de } \\
\text { software são fundamentais na AR. }\end{array}$ \\
\hline QP 2.7 & $\begin{array}{l}\text { Como cada AR lida com a } \\
\text { interoperabilidade de seus } \\
\text { constituintes? }\end{array}$ & $\begin{array}{l}\text { Identificar métodos e técnicas usadas nas ARs para } \\
\text { garantir interoperabilidade. }\end{array}$ \\
\hline QP 3. & $\begin{array}{l}\text { Qual o principal tipo de } \\
\text { contribuição do estudo? }\end{array}$ & $\begin{array}{l}\text { Identificar as contribuições existentes no contexto de } \\
\text { SoS. (p.ex. processo, método, modelo, arcabouço, } \\
\text { ferramenta, aplicação, métrica, arquitetura, etc.) }\end{array}$ \\
\hline QP 4. & $\begin{array}{l}\text { Quais direções futuras para } \\
\text { a pesquisa? }\end{array}$ & $\begin{array}{l}\text { Esta questão foi elaborada para extrair as direções de } \\
\text { pesquisa mencionadas pelos estudos incluídos em suas } \\
\text { respectivas conclusões. }\end{array}$ \\
\hline
\end{tabular}

\subsection{Estratégia de Busca}

As seguintes palavras-chave e seus sinônimos foram obtidos a partir das publicações Guessi et al. (2010) e Nakagawa et al. (2017), e com a colaboração de especialistas na área de SoS:

- Reference Architecture (Conceptual Architecture, Reference Model);

- System of Information System (SoIS, Systems of Information Systems, Systems- 
of-Information Systems);

- System of System (SoS, System of System, Systems of Systems, Systems-ofSystems).

Os termos SoS e SoIS foram usados em conjunto para atingir uma maior abrangência, cobrindo os SoS e forçando o retorno de estudos que eventualmente tratem de SoIS em particular. Com base nas palavras-chave e seus sinônimos, foi criada uma primeira versão da string de busca para realização do teste piloto. Inicialmente, o termo "conceptual architecture" foi aplicado no primeiro braço da string. Entretanto, a base de publicações digital ACM (Association for Computing Machinery) retornou muitos artigos não relacionados ao tópico de interesse e o IEEE (Institute of Electrical and Electronics Engineers) não apresentou alteração quanto à quantidade de estudos retornados. $\mathrm{O}$ termo "constituent" também foi testado sem nenhuma alteração nos resultados. A string de busca foi ajustada após vários testes nas bases escolhidas e assumiu a seguinte forma:

("reference architecture" OR "reference model") AND ("Systems-of-Systems" OR "Systems of Systems" OR "System of Systems" OR "SoS" OR "Systems of Information Systems" OR "Systems-of-Information Systems" OR "SoIS")

Com a string de busca ajustada, a pesquisa final foi realizada no dia 27 de setembro de 2019 em todas as bases de publicações digitais selecionadas, posteriormente foi realizada uma atualização das buscas no dia 11 de fevereiro de 2021. A busca realizada foi automática, sem filtro temporal e aplicada aos metadados. As seguintes fontes foram utilizadas:

- ACM DL, (The ACM Guide to Computing Literature),

- Engineering Village,

- IEEE Xplore,

- Scopus,

- ScienceDirect.

Os critérios para a seleção destas fontes de pesquisa foram os seguintes:

- fontes consideradas relevantes para a área de Sistemas de Sistemas;

- fontes com mecanismo de busca disponível na Web e com suporte a expressões booleanas;

- fontes que possibilitem busca sobre metadados, no mínimo, sobre o resumo/abstract;

- fontes que exportem resultados de busca para múltiplos formatos com acesso disponível pelas principais ferramentas de apoio ao mapeamento sistemático.

A quantidade de estudos retornados por cada base é apresentada na Tabela 2.

Tabela 2. Quantidade de Estudos Retornados

\begin{tabular}{|l|l|}
\hline Fontes & $\begin{array}{l}\text { Estudos } \\
\text { Encontrados }\end{array}$ \\
\hline
\end{tabular}




\begin{tabular}{|l|l|}
\hline ACM DL & 99 \\
\hline Engineering Village & 101 \\
\hline IEEE DL & 53 \\
\hline Science Direct & 2 \\
\hline Scopus & 106 \\
\hline
\end{tabular}

Foram definidos também critérios para incluir e excluir estudos do mapeamento. Se o estudo atende ao critério de inclusão (CI), ele é aceito. Analogamente, caso ele atenda a quaisquer dos critérios de exclusão (CE), ele é eliminado. O CI definido foi o seguinte:

- O estudo trata de arquiteturas ou modelos de referência de software para SoS ou SoIS.

Foram definidos os seguintes CEs:

- CE1 - Não é um estudo primário;

- CE2 - Não é um artigo publicado em anais de eventos ou periódicos, isto é, o estudo não é selecionado via procedimento de revisão por pares;

- CE3 - Texto completo não se encontra na língua portuguesa e/ou inglesa;

- CE4 - Texto completo não está disponível para acesso;

- CE5 - Artigo cujo tema é Arquitetura de Referência, mas não aplica-se a SoS/SoIS;

- CE6 - Artigo cujo tema é SoS, mas não trata de arquiteturas ou modelos de referência;

- CE7 - Artigo cujo tema é modelo de referência, mas não trata arquitetura de software;

- CE8 - Estudo é uma versão mais antiga de outro estudo já considerado.

A ferramenta Parsifal ${ }^{1}$ foi utilizada como apoio ao desenvolvimento do mapeamento. Após cadastrar na ferramenta os estudos retornados de cada fonte de busca via busca automática, a seguinte estratégia de seleção foi adotada:

- Identificar e remover estudos duplicados;

- Com base na leitura do título, resumo e palavras-chave de cada estudo nãoduplicado, classificá-lo como aceito ou rejeitado de acordo com os critérios de inclusão ou exclusão. Havendo dúvida na inclusão ou exclusão de um estudo, um especialista foi consultado para o veredito final. Quando o consenso não foi

$1 \quad$ https://parsif.al/

iSys: Revista Brasileira de Sistemas de Informação (iSys: Brazilian Journal of Information Systems) https://sol.sbc.org.br/journals/index.php/isys 
atingido, o estudo foi incluído para leitura do texto completo;

- Com base na leitura do texto completo dos estudos aceitos, procedeu-se a classificá-lo novamente pelos critérios de inclusão ou exclusão.

A partir do conjunto final de estudos do mapeamento sistemático, os dados importantes foram extraídos e sintetizados. Para auxiliar nesta tarefa, um formulário de extração foi criado utilizando a ferramenta Parsifal. O Apêndice 1 apresenta as questões contidas neste formulário e o mapeamento delas para as questões de pesquisa elaboradas.

Após a extração das informações relevantes com o formulário, uma síntese narrativa foi desenvolvida com o intuito de apresentar o resultado obtido com o mapeamento sistemático.

\subsection{Condução}

A próxima etapa do processo de mapeamento sistemático consistiu na seleção dos estudos para extração dos dados. O mapeamento foi conduzido entre Agosto de 2018 e Março de 2021. Duas rodadas de buscas foram realizadas com a mesma chave e nas mesmas bases. A primeira rodada aconteceu em Setembro de 2018 e uma nova busca foi executada em Janeiro de 2021 a título de atualização do mapeamento. Inicialmente, 20 estudos haviam sido incluídos. A partir da atualização, 3 novos estudos foram incluídos, totalizando 23 estudos. A Figura 2 apresenta uma síntese com o resultado quantitativo de estudos em cada etapa do processo de mapeamento sistemático. Foram retornados 361 estudos nas duas buscas automáticas conduzidas. Após a exclusão dos estudos duplicados (148 estudos), restaram um total de 213 estudos para análise nas etapas seguintes.

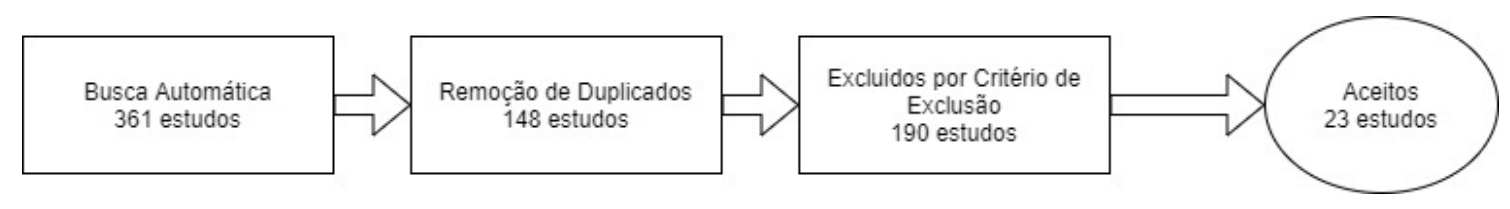

Figura 2. Número de Estudos por Etapa.

Como apenas um CI referente à necessidade de os estudos abordarem arquiteturas de referência para SoS ou SoIS foi definido, todos os 23 estudos aceitos atendem a tal CI. Entretanto, os 190 estudos rejeitados foram distribuídos pelos CEs. A Figura 3 apresenta a quantidade de estudos que foram excluídos por atender a cada CE específico. 


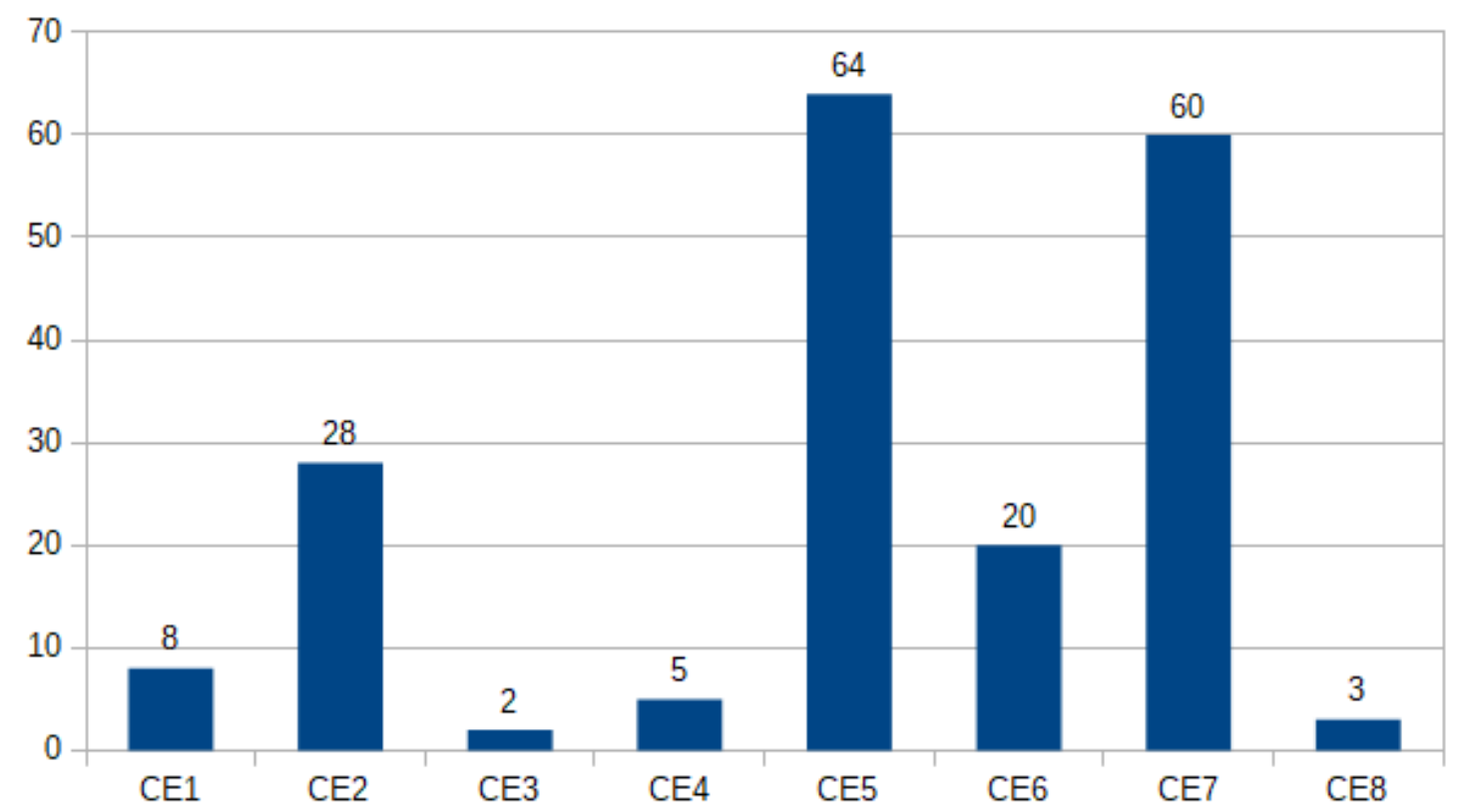

Figura 3. Número de Estudos Excluídos por CE.

A Tabela 3 apresenta um mapeamento dos estudos obtidos e excluídos por base de busca neste mapeamento sistemático.

Tabela 3. Aproveitamento de Estudo por Base.

\begin{tabular}{|l|c|c|c|c|}
\hline Fonte & Encontrados & Rejeitados & Duplicados & Aceitos \\
\hline ACM & 99 & 85 & 11 & $\mathbf{3}$ \\
\hline $\begin{array}{l}\text { El } \\
\text { Compendex }\end{array}$ & 101 & 38 & 56 & $\mathbf{7}$ \\
\hline IEEE & 53 & 16 & 34 & $\mathbf{3}$ \\
\hline $\begin{array}{l}\text { Science } \\
\text { Direct }\end{array}$ & 2 & 2 & 0 & $\mathbf{0}$ \\
\hline Scopus & 106 & 50 & 46 & $\mathbf{1 0}$ \\
\hline
\end{tabular}

Após a análise dos estudos e aplicação dos critérios de inclusão e exclusão, $\underline{\mathbf{2 3}}$ estudos foram incluídos. Na Tabela 4, são apresentados os estudos selecionados com seus respectivos identificadores.

Tabela 4. Estudos Selecionados

\begin{tabular}{|c|c|l|l|}
\hline ID & Ano & \multicolumn{1}{|c|}{ Título } & \multicolumn{1}{|c|}{ Autores } \\
\hline S1 & 2005 & $\begin{array}{l}\text { Assured counter-terrorism information } \\
\text { sharing using Attribute Based Information } \\
\text { Security (ABIS) }\end{array}$ & Yuan, E. and Wenzel, G. \\
\hline
\end{tabular}




\begin{tabular}{|c|c|c|c|}
\hline S2 & 2008 & $\begin{array}{l}\text { A method for collaborative development of } \\
\text { systems of systems in the office domain }\end{array}$ & $\begin{array}{l}\text { Carbon, R. and Johann, G. } \\
\text { and Muthig, D. and Naab, M. }\end{array}$ \\
\hline S3 & 2008 & $\begin{array}{l}\text { A framework of municipal management } \\
\text { information systems (MMIS) in e-government }\end{array}$ & $\begin{array}{l}\text { Wang, Yuanfang and Zhou, } \\
\text { Hongren and Jing, } \\
\text { Zhongliang }\end{array}$ \\
\hline S4 & 2009 & $\begin{array}{l}\text { Army enterprise architecture technical } \\
\text { reference model for system interoperability }\end{array}$ & $\begin{array}{l}\text { Zhu, S. T. and Wong, R. W. } \\
\text { and McDonough, C. A. and } \\
\text { Roy, R. R. and Fine, J. M. } \\
\text { and Reiling, J. P. }\end{array}$ \\
\hline S5 & 2009 & $\begin{array}{l}\text { Managing systems of systems } \\
\text { interoperability - federated soa and reference } \\
\text { architectures }\end{array}$ & $\begin{array}{l}\text { Chang, C. F. and Tan, P. H. } \\
\text { and Tam, S. K. }\end{array}$ \\
\hline S6 & 2011 & $\begin{array}{l}\text { Model Based Systems Engineering for Smart } \\
\text { Grids as systems of systems }\end{array}$ & $\begin{array}{l}\text { Lopes, A. J. and Lezama, R. } \\
\text { and Pineda, R. }\end{array}$ \\
\hline S7 & 2013 & $\begin{array}{l}\text { Improving disaster management using earth } \\
\text { observations-GEOSS and CEOS activities }\end{array}$ & $\begin{array}{l}\text { Percivall, G. S. and Alameh, } \\
\text { N. S. and Caumont, H. and } \\
\text { Moe, K. L. and Evans, J. D. }\end{array}$ \\
\hline S8 & 2014 & $\begin{array}{l}\text { Strengthening Architectures of Smart CPS } \\
\text { by Modeling Them As Runtime Product-lines }\end{array}$ & $\begin{array}{l}\text { Bures, T. and Hnetynka, P. } \\
\text { and Plasil, F. }\end{array}$ \\
\hline S9 & 2015 & $\begin{array}{l}\text { Towards a Reference Architecture for Large- } \\
\text { scale Smart Grids System of Systems }\end{array}$ & $\begin{array}{l}\text { Pérez, J. and Díaz, J. and } \\
\text { Garbajosa, J. and Yagüe, A. } \\
\text { and Gonzalez, E. and Lopez- } \\
\text { Perea, M. }\end{array}$ \\
\hline S10 & 2016 & $\begin{array}{l}\text { Using the view model to contextualize and } \\
\text { explain system-of-systems architecture } \\
\text { models }\end{array}$ & $\begin{array}{l}\text { Walewski, J. W. and Heiles, } \\
\text { J. }\end{array}$ \\
\hline S11 & 2016 & $\begin{array}{l}\text { SGAM business layer for a local flexibility } \\
\text { market }\end{array}$ & $\begin{array}{l}\text { Pavlovic, M. and Gawron- } \\
\text { deutsch, T. and Neureiter, C. } \\
\text { and Diwold, K. }\end{array}$ \\
\hline S12 & 2016 & $\begin{array}{l}\text { ITE arbitrator: A reference architecture } \\
\text { framework for sustainable IT ecosystems }\end{array}$ & Park, S. and Park, Y.B. \\
\hline S13 & 2016 & $\begin{array}{c}\text { A standards-based approach for domain } \\
\text { specific modelling of smart grid system } \\
\text { architectures }\end{array}$ & $\begin{array}{l}\text { Neureiter, C. and Uslar, M. } \\
\text { and Engel, D. and Lastro, G. }\end{array}$ \\
\hline
\end{tabular}




\begin{tabular}{|c|c|c|c|}
\hline $\mathrm{S} 14$ & 2017 & $\begin{array}{c}\text { Local Cloud Internet of Things Automation: } \\
\text { Technology and Business Model Features of } \\
\text { Distributed Internet of Things Automation } \\
\text { Solutions }\end{array}$ & Delsing, J. \\
\hline S15 & 2017 & $\begin{array}{c}\text { Agile digital transformation of System-of- } \\
\text { Systems architecture models using } \\
\text { Zachman framework }\end{array}$ & $\begin{array}{l}\text { Bondar, S. and Hsu, J.C. } \\
\text { and Pfouga, A. and } \\
\text { Stjepandić, J. }\end{array}$ \\
\hline S16 & 2017 & $\begin{array}{c}\text { A process to establish, model and validate } \\
\text { missions of systems-of-systems in reference } \\
\text { architectures }\end{array}$ & $\begin{array}{c}\text { Garces, L. and Nakagawa, E. } \\
\text { Y. }\end{array}$ \\
\hline S17 & 2017 & $\begin{array}{l}\text { Using a systems of systems modeling } \\
\text { approach for developing Industrial Internet } \\
\text { of Things applications }\end{array}$ & $\begin{array}{l}\text { Morkevicius, A. and } \\
\text { Bisikirskiene, L. and } \\
\quad \text { Bleakley, G. }\end{array}$ \\
\hline $\mathrm{S} 18$ & 2018 & $\begin{array}{c}\text { Towards software architectural description } \\
\text { for systems of systems: Case of a maritime } \\
\text { transport SoS }\end{array}$ & $\begin{array}{c}\text { Seghiri, A. and Belala, F. and } \\
\text { Teniou, A.A. }\end{array}$ \\
\hline $\mathrm{S} 19$ & 2018 & $\begin{array}{l}\text { An Internet of Things system architecture for } \\
\text { aiding firefighters in the scene of disaster }\end{array}$ & $\begin{array}{l}\text { Lee, H. and Hong, S.G. and } \\
\text { Lee, K.B. }\end{array}$ \\
\hline $\mathrm{S} 20$ & 2019 & $\begin{array}{l}\text { Autonomous driving as system of systems: } \\
\text { Roadmap for accelerating development }\end{array}$ & $\begin{array}{l}\text { Assaad, M. A. and Talj, R. } \\
\text { and Charara, A. }\end{array}$ \\
\hline S21 & 2020 & $\begin{array}{l}\text { On the Engineering of loT-Intensive Digital } \\
\text { Twin Software Systems }\end{array}$ & $\begin{array}{l}\text { Rivera, L. F. and Müller, H. A. } \\
\text { and Villegas, N. M. and } \\
\text { Tamura, G. and Jiménez, M. }\end{array}$ \\
\hline S22 & 2020 & $\begin{array}{l}\text { A design of the resilient enterprise: } A \\
\text { reference architecture for emergent } \\
\text { behaviors control }\end{array}$ & $\begin{array}{l}\text { Bemthuis, R. and lacob, M. } \\
\text { and Havinga, P. }\end{array}$ \\
\hline $\mathrm{S} 23$ & 2020 & $\begin{array}{l}\text { Beyond connected cars: A systems of } \\
\text { systems perspective }\end{array}$ & $\begin{array}{l}\text { Pelliccione, P. and Knauss, } \\
\text { E. and Ågren, S.M. and } \\
\text { Heldal, R. and Bergenhem, } \\
\text { C. and Vinel, A. and } \\
\text { Brunnegård, O. }\end{array}$ \\
\hline
\end{tabular}

\subsection{Resultados}

A partir da extração e síntese dos resultados, as questões de pesquisa puderam ser respondidas, como consta a seguir.

\subsubsection{QP 1. Quando e em quais veículos de divulgação científica os estudos têm sido publicados?}


A Figura 4 apresenta a distribuição dos estudos incluídos ao longo do tempo. As publicações analisadas estão distribuídas entre os anos de 2005 e 2020. Como o levantamento sistemático foi realizado no mês de fevereiro de 2021, podem existir estudos relevantes publicados após esta data que não foram indexados e incluídos. Notase que a maior quantidade de estudos incluídos foram publicados entre os anos de $2016 \mathrm{e}$ 2017. Ainda que haja alguma oscilação, nota-se uma ascensão no número de estudos tratando do tópico ao longo dos anos. Tal indício mostra que a aplicação de ARs no contexto de SoS tem recebido interesse e tem sido considerada relevante. Produtos de software cuja arquitetura foi derivada de uma AR costumam exigir elevado grau de qualidade devido à uniformização e reúso trazidos pelas ARs. O aumento no número de estudos sugere que os pesquisadores de SoS podem ter se motivado a usar AR no desenvolvimento de SoS nos últimos anos para tentar alcançar resultados semelhantes ao que ocorre em sistemas de software isolados.

O veículo com mais publicações dentre as analisadas foi a Conference System of Systems Engineering (SoSE) com 17,4\% da publicações (4 estudos, S10, S13, S17, S20) seguido pelo International Workshop on Software Engineering for Systems-of-Systems (SESoS) com 8,7\% (2 estudos, S9, S12). Os demais estudos foram publicados em veículos diferentes como pode ser visto no Apêndice 2 . Se for considerado o tipo de publicação, 69,6\% foram em eventos, tais como congressos simpósios, conferências e $30,4 \%$ em periódicos, o que deixa mais evidente o fato de ser uma área ainda pouco explorada e que precisa de maior amadurecimento e robustez nos resultados experimentais.

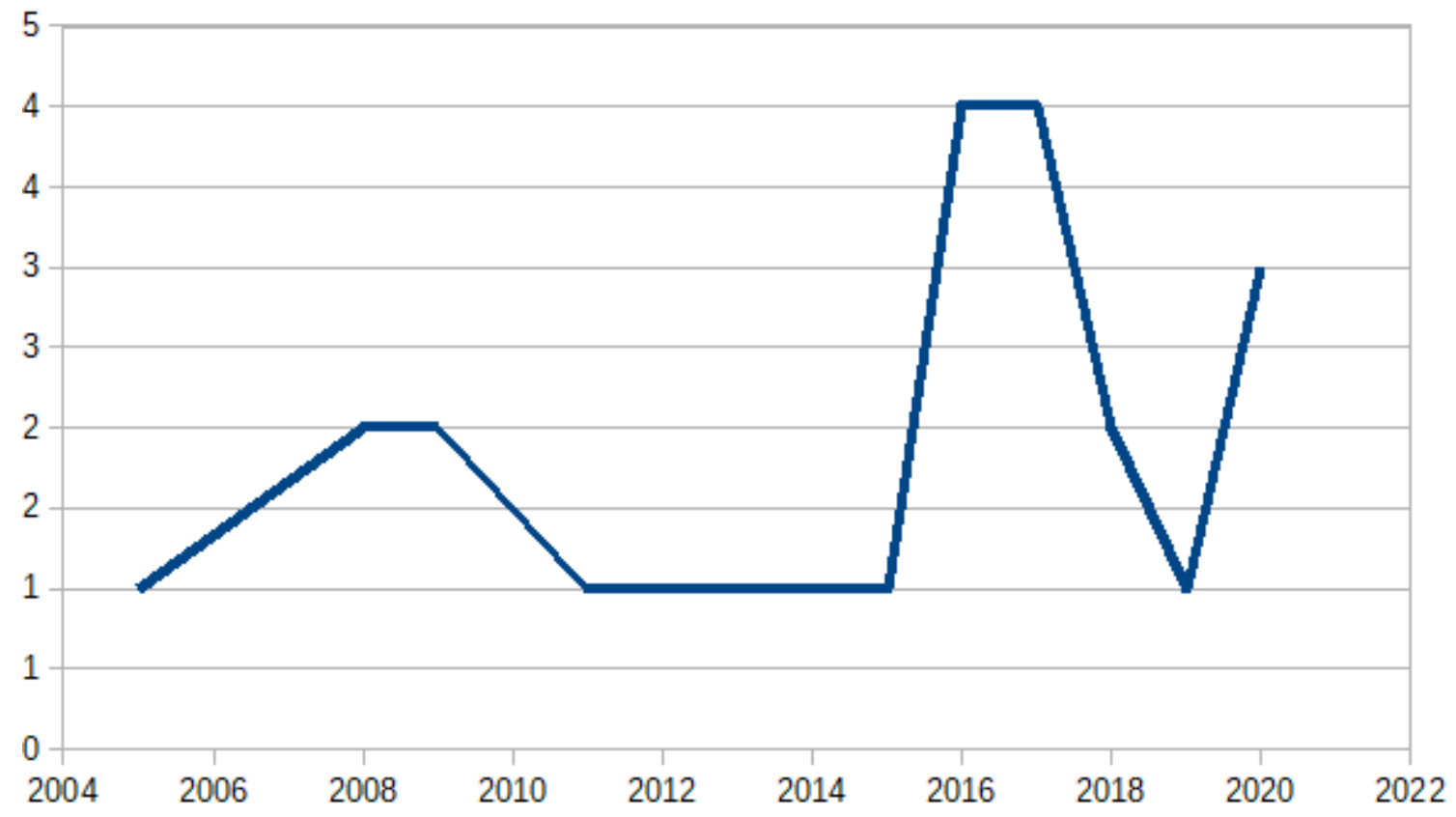

Figura 4. Gráfico dos estudos publicados no decorrer dos anos.

3.4.2. QP 2. Quais arquiteturas de referência têm sido propostas no contexto de SoS/SoIS?

Para responder a esta questão de pesquisa, foi necessário desmembrá-la em sete subquestões. 
QP 2.1. Quais modelos de referência ou métodos são usados para criar arquitetura de referência?

As informações que caracterizam as ARs apresentadas foram coletadas utilizando o formulário disponível no Apêndice. A Tabela 5 mostra a lista de arquiteturas, modelos de referência e processos de concepção relatados nos estudos incluídos. Nos estudos selecionados, há uma grande diversidade de formas de representação arquitetural. Nem todos os estudos relatam o uso de métodos de modelagem como base de suas ARs, mas também normas e arquiteturas. Apenas os estudos S5, S7, S12 e S22 não apresentam o método que foi utilizado na concepção arquitetural. Por outro lado, o estudo S20 apresenta um roteiro das atividades que devem ser seguidas para a criação das arquiteturas de referências específicas no domínio de transportes. Os estudos S1, S8 e S10 também propõem métodos para a criação das ARs. O estudo S3 relata o uso do E-government Reference Models (ERM) como base para produzir sua AR. ERM é um modelo já existente e específico utilizado para o caso de Xangai, que trata-se de um plano piloto de implementação do e-governo em Xangai. O estudo S2 utilizou como base o PuLSE (Product Line Software and Systems Engineering). O estudo S9 utiliza como referência a Arquitetura Baseada em Serviços e Orientada a Eventos (EDSOA).

Tabela 5. Modelos de Referência/Método dos Estudos.

\begin{tabular}{|c|c|}
\hline Modelo/Método & Estudos \\
\hline $\begin{array}{c}\text { ABIS (Attribute } \\
\text { Based Information } \\
\text { Security) }\end{array}$ & S1 \\
\hline $\begin{array}{l}\text { PuLSE (Product Line } \\
\text { Software and } \\
\text { Systems Engineering) }\end{array}$ & S2 \\
\hline $\begin{array}{l}\text { ERM (E-government } \\
\text { Reference Models) }\end{array}$ & S3 \\
\hline FEA TRM & S4 \\
\hline $\begin{array}{c}\text { NIST Logical } \\
\text { Reference Model }\end{array}$ & $\mathrm{S} 6, \mathrm{~S} 13$ \\
\hline SOFA NG & S8 \\
\hline $\begin{array}{l}\text { EDSOA (Arquitetura } \\
\text { Baseada em } \\
\text { Serviços e Orientada } \\
\text { a Eventos) }\end{array}$ & S9 \\
\hline $\begin{array}{l}\text { MDA (Model Driven } \\
\text { Architecture) }\end{array}$ & S11 \\
\hline
\end{tabular}




\begin{tabular}{|c|c|}
\hline RAMI 4.0 & $\mathrm{S} 10, \mathrm{~S} 14$ \\
\hline $\begin{array}{c}\text { Framework de } \\
\text { Zachman }\end{array}$ & $\mathrm{S} 15$ \\
\hline ProSA-RA & $\mathrm{S} 16$ \\
\hline $\begin{array}{c}\text { UAF (United } \\
\text { Architecture } \\
\text { Framework) }\end{array}$ & $\mathrm{S} 17$ \\
\hline RAModel & $\mathrm{S} 18$ \\
\hline $\begin{array}{c}\text { SPL (Engenharia de } \\
\text { Linhas de Produto de } \\
\text { Software) }\end{array}$ & $\mathrm{S} 19$ \\
\hline ACRA & $\mathrm{S} 21$ \\
\hline $\begin{array}{c}\text { ASO/IEC/IEEE 42010 } \\
\text { Não Apresenta }\end{array}$ & $\mathrm{S} 5, \mathrm{~S} 7, \mathrm{~S} 12, \mathrm{~S} 22$ \\
\hline
\end{tabular}

Os estudos S6 e S13 utilizam como referência o NIST Logical Reference Model, que é um modelo conceitual associado a uma guideline que contém uma coleção de abordagens para soluções em cenários de smart grid. Por sua vez, os estudos S15 e S17 usam frameworks arquiteturais como base para estabelecer arquiteturas em SoS. O S15 usa o Framework de Zachman e o S17 usa como referência o United Architecture Framework (UAF). O estudo S16 utilizou o processo ProSA-RA para criar uma AR para a modelagem e validação de missões no contexto de SoS. O S14 trata do uso de uma AR chamada RAMI 4.0 para representar a arquitetura de um SoS no contexto industrial. O S11 tem como base a MDA (Model Driven Architecture) e utiliza esta abordagem no contexto de smart grid para distribuição local de energia. O estudo S19 tem como base o uso de Engenharia de Linhas de Produto de Software para a concepção de uma AR de SoS no contexto de combate a incêndios. O S18 trouxe o RA-SoS, que é uma AR específica para SoS. Essa AR foi proposta com base no RAModel e foi concebida para arquitetura do SoS como um todo no domínio de transporte marítimo. O estudo S21, por sua vez, baseou a concepção de sua AR na arquitetura de referência ACRA e no modelo de referência DYNAMICO. Por fim, o estudo S23 propôs uma AR para carros conectados utilizando a ISO/IEC/IEEE 42010 para fazer a descrição de sua arquitetura.

Neste aspecto foi encontrada uma variedade de modelos utilizados para direcionar a criação das arquiteturas de referência, como apresentado na seção anterior. Na maioria dos trabalhos analisados, fica evidente que os modelos usados para a criação das ARs não são específicos para este fim. Alguns estudos (S2, S4, S9, S15, S17, S19, S23 que totalizam $30,4 \%$ do total de estudos analisados) indicam apenas a utilização de arquiteturas reais, frameworks e normas técnicas. 52,2\% dos estudos (S1, S3, S6, S8, S10, 
S11, S13, S14, S16, S18, S20, S21) usam, de fato, ARs ou modelos de referência para a concepção de suas arquiteturas. O demais não apresentaram em que se basearam (S5, S7, S12, S22, que são $17,4 \%$ do total)

Portanto, pode-se concluir que existem diversas possibilidades para criar uma arquitetura de referência, tais como os modelos propostos SOFA NG e NIST e o RA-SoS inspirado no RAModel. Além dos modelos propostos, também foram utilizados frameworks, modelos de referência, processos e normas técnicas.

QP 2.2. Para quais domínios elas têm sido propostas?

Em relação ao domínio de aplicação, os domínios energético (smart grid) e de segurança física lideram nos estudos com o desenvolvimento de 21,7\% das arquiteturas SoS. Outros trabalhos apresentam propostas de arquitetura SoS nos domínios de transporte $(17,4 \%)$ e da indústria $(13 \%)$. As áreas de segurança da informação, SoS de escritório, governo, CPS (Smart Cyber-Physical Systems), comércio e saúde também aparecem nos artigos selecionados. A Tabela 6 apresenta os estudos e sua relação com a área de aplicação.

Tabela 6. Domínio de Aplicação dos Estudos.

\begin{tabular}{|c|c|}
\hline Domínio & Estudos \\
\hline Segurança da Informação & S1 \\
\hline Escritório & S2 \\
\hline Governo & S3 \\
\hline CPS & S8 \\
\hline Smart Grid & $\begin{array}{c}\text { S6, S9, } \\
\text { S11, S13, } \\
\text { S14 }\end{array}$ \\
\hline Segurança & $\begin{array}{l}\text { S4, S5, } \\
\text { S7, S12, } \\
\text { S19 }\end{array}$ \\
\hline Transporte & $\begin{array}{l}\text { S18, S20, } \\
\text { S21, S23 }\end{array}$ \\
\hline Indústria & $\begin{array}{l}\text { S10, S17 } \\
\text { S22 }\end{array}$ \\
\hline Comércio & S15 \\
\hline Saúde & S16 \\
\hline
\end{tabular}

De forma mais específica, o estudo S1 apresenta a segurança da informação no 
contexto de terrorismo. Os estudos S4, S5, S7, S12 e S19 também apresentam ARs no domínio de segurança, sendo os dois primeiros em sistemas militares e de defesa. Os estudos S7 e S19 apresentam ARs para sistemas de prevenção de desastres e o S12 para gerenciamento florestal. A prevenção de desastres focada no estudo S19 tem como principal função a atividade dos bombeiros e sua segurança em ação, já o S7 em desastres ambientais. A Tabela 7 resume os domínios de segurança específicos de cada estudo.

Tabela 7. Domínio Específico de Segurança
\begin{tabular}{|l|l|}
\hline Domínio Específico & \multicolumn{1}{|c|}{ Estudos } \\
\hline Segurança da Informação & S1 \\
\hline Sistema Militar & S4 e S5 \\
\hline Gerenciamento Florestal & S12 \\
\hline Combate a Incêndio & S19 \\
\hline $\begin{array}{l}\text { Combate a Desastres } \\
\text { Ambientais }\end{array}$ & S7 \\
\hline
\end{tabular}

O estudo S2 apresenta a AR para no contexto de escritório de modo a ter uma base para que os softwares do escritório possam formar um SoS. No estudo S3, a AR está no contexto do governo municipal de Xangai.

A área de energia é amplamente discutida em alguns dos estudos incluídos. $\mathrm{O}$ estudo A6 apresenta um modelo arquitetural para a área de engenharia. O S9 apresenta uma AR para smart grids de larga escala. O S11 apresentou um modelo SGAM (Smart Grid Architecture Model) e o S13 aplica o modelo na área de matriz energética. O estudo S14 relata a especificação de uma arquitetura de referência de internet das coisas com aplicação em smart grid. Na Tabela 8, os subdomínios da aplicação em smart grid são apresentados resumidamente.

Tabela 8. Domínio Específico de Smart Grid
\begin{tabular}{|l|l|}
\hline Domínio Específico & \multicolumn{1}{|c|}{ Estudos } \\
\hline Engenharia & S6 \\
\hline Larga Escala & S9 \\
\hline Modelo Arquitetural & S11 e S13 \\
\hline Internet das Coisas & S14 \\
\hline
\end{tabular}

O estudo A8 trata de sistemas cyber físicos. O S10, S17 e o S22 estão focados na indústria (processos industriais). O S18 está no domínio do transporte marítimo. Por sua vez, os estudos S20 e o S23 também estão no domínio do transporte só que terrestre e com foco em carros inteligentes e autônomos. O S21 tem seu foco no transporte público.

Já o S15 está no domínio do comércio, a sua atuação foi voltada à cadeia de suprimentos automotivos. O domínio da saúde teve o S16 como único representante com um processo sistemático para estabelecer, modelar e validar missões. 
Dentre os estudos analisados, nota-se maior foco nos domínios de smart grid e segurança pública. Os domínios de automação industrial e transporte também tiveram boa representatividade nos estudos analisados. Nota-se ainda que existem muitos domínios a serem explorados no âmbito de arquiteturas de referência para SoS.

QP 2.3. Quais estilos arquiteturais têm sido utilizados nas ARs?

Em relação ao estilo arquitetural, $60,9 \%$ dos estudos utilizava o estilo em camadas. Os estudos incluídos utilizam o estilo Camadas para descrever a arquitetura do SoS como um todo, dividindo os níveis de controle e abstração do SoS em camadas. Exemplos de camadas encontradas nos estudos são camadas de gerenciamento, conexão e orquestração. O estudo S6, por exemplo, recomenda o uso de camadas de controle e camada de serviço. As camadas são usadas para dividir o SoS como um todo em níveis de atuação detalhando seu funcionamento. O estudo $\mathrm{S} 4$, por sua vez, relata o uso de um estilo centrado em base de dados $(4,3 \%)$. Não houve estudos que utilizassem estilos arquiteturais para tratarem da arquitetura interna de um constituinte, por exemplo. Por outro lado, 34,8\% dos estudos não deixam evidente o estilo arquitetural adotado. A Tabela 9 apresenta quais estudos adotam ou não um estilo arquitetural.

Tabela 9. Estilo Arquitetural utilizado nos Estudos.

\begin{tabular}{|c|c|}
\hline $\begin{array}{c}\text { Estilo } \\
\text { Arquitetural }\end{array}$ & Estudos \\
\hline Camadas & $\begin{array}{c}\text { S3, S5, S6, S8, S9, } \\
\text { S10, S11, S12, S13, } \\
\text { S14, S15, S17, S21, } \\
\text { S22 }\end{array}$ \\
\hline $\begin{array}{c}\text { Centrado em } \\
\text { Base de Dados }\end{array}$ & S4 \\
\hline Não especifica & $\begin{array}{c}\text { S1, S2, S7, S16, S18, } \\
\text { S19, S20, S23 }\end{array}$ \\
\hline
\end{tabular}

Uma possível razão para a prevalência do estilo arquitetural em camadas no contexto de ARs para SoS deve-se à compatibilidade entre a estrutura e comportamento prescritos pelo estilo arquitetural Camadas e as necessidades inerentes à engenharia de SoS. O estilo de Camadas é conhecido por permitir a modularização dos interesses de um sistema em diferentes graus de abstração e de modo que camadas superiores possam recorrer a serviços disponíveis em camadas subjacentes. No que tange aos SoS, é plausível conceber a arquitetura do sistema em níveis de abstração diferentes, tal como apresentado nos estudos S9 e S12 e como mostramos na Figura 5 e discutimos a seguir. Em uma estrutura em camadas, os níveis inferiores podem lidar com aspectos mais técnicos, tais como a persistência dos dados (ainda que internos aos próprios constituintes) e detalhes de comunicação necessários para a devida interoperabilidade entre os constituintes. Os níveis superiores de abstração, por sua vez, encapsulam os componentes de software dos constituintes bem como suas funcionalidades, que apoiamse nas infraestruturas de comunicação para a operação do SoS. Numa camada ainda 
superior à software, são tratadas questões gerenciais e de negócio, apoiando-se em todos os insumos de dados e trocas de informações oferecidos pelas infra-estruturas de software e hardware/redes subjacentes. Ao que parece, o modelo de camadas acomoda de forma mais bem sucedida os diferentes níveis de abstração inerentes ao SoS que, através de seus constituintes, são estratificados em camadas de abstração bem definidas.

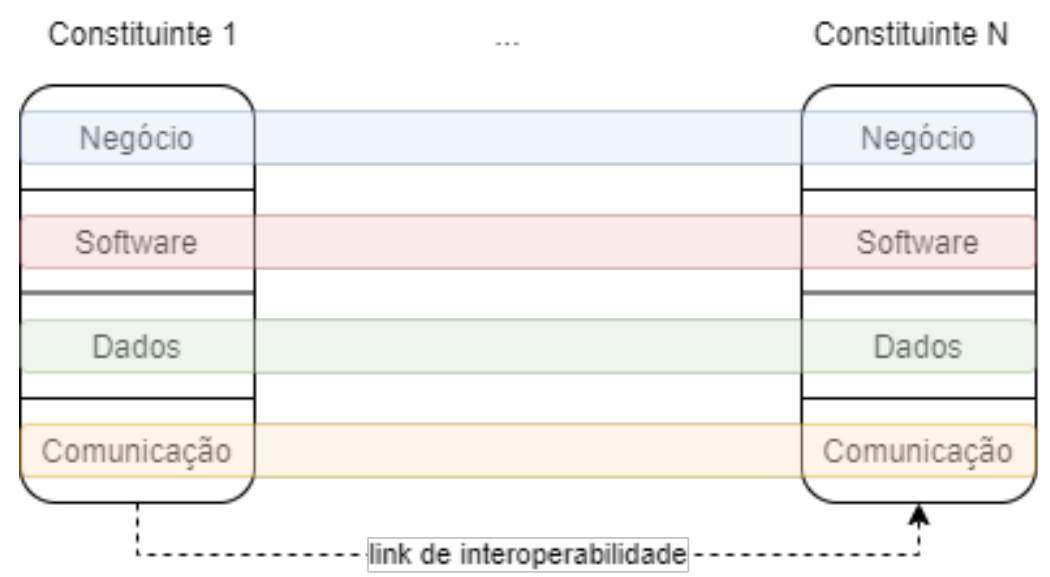

Figura 5. Conceituação de camadas no nível do SoS.

QP 2.4. Quais métodos são usados para avaliar as ARs?

A Tabela 10 apresenta a classificação dos estudos conforme o tipo de avaliação aplicada. 65,2\% dos estudos apresentaram e discutiram a abordagem de prova de conceito, isto é, mostrar que era possível derivar uma arquitetura concreta aderente à AR proposta. Dentre os demais estudos, S1 e S12 $(8,7 \%)$ utilizaram simulação para avaliar arquiteturas concretas derivadas de uma AR proposta para o SoS, enquanto os estudos S11 e S23 utilizaram consulta com especialistas para auxiliar na avaliação da arquitetura quanto às características funcionais e não funcionais $(8,7 \%)$. O estudo S19 apresentou um experimento controlado. Os estudos S3, S4, S5, S6 e S7, por sua vez, utilizaram a abordagem de Pesquisa-ação para a condução de estudos de caso em ambiente real.

Tabela 10. Avaliação das Arquiteturas.

\begin{tabular}{|c|c|}
\hline Avaliação & Estudos \\
\hline Prova de Conceito & $\begin{array}{c}\text { S2, S8, S10, S13, } \\
\text { S14, S16, S17, } \\
\text { S21, S22 }\end{array}$ \\
\hline Experimento Controlado & $\mathrm{S} 19$ \\
\hline Pesquisa-Ação & $\mathrm{S} 3, \mathrm{~S} 4, \mathrm{~S} 5, \mathrm{~S} 6, \mathrm{~S} 7$ \\
\hline Simulação & $\mathrm{S} 1, \mathrm{~S} 12$ \\
\hline
\end{tabular}




\begin{tabular}{|c|c|}
\hline Entrevista com Especialista & $\mathrm{S} 11, \mathrm{~S} 23$ \\
\hline Não apresenta & $\mathrm{S} 6, \mathrm{~S} 9, \mathrm{~S} 18, \mathrm{~S} 20$ \\
\hline
\end{tabular}

Nota-se que, embora existam métodos que indiquem a utilização de questionários com as partes interessadas ou consulta com especialistas, a prova de conceito parece ser ainda a forma mais popular de avaliar uma arquitetura de referência, sendo por isso mais usada.

QP 2.5. Quais as tecnologias recomendadas/prescritas nestas arquiteturas?

A Tabela 11 apresenta as tecnologias citadas nos estudos. O estudo S9 é o único que recomenda, explicitamente, algumas tecnologias para uso nas camadas utilizadas na AR. O estudo recomenda, por exemplo, o uso de Java Messaging Service (JMS) para comunicação e NoSQL na camada de Dados. Mais da metade dos trabalhos $(65,2 \%)$ analisados não indica uma tecnologia para o desenvolvimento de ARs. Os estudos não recomendam explicitamente o uso de tecnologias de desenvolvimento de software que poderiam, por exemplo, ser usadas para configurar um ambiente de implantação de uma arquitetura concreta derivada de uma AR. Por outro lado, a maioria dos estudos recomenda o uso de tecnologias específicas de redes para lidar com o atributo de conectividade. Os estudos S4, S8 e S14 recomendam o uso de padrões de área, isto é, se a arquitetura é voltada para conectividade em rede, então deve-se utilizar os protocolos específicos daquele tipo de rede disponível, tais como IEEE 802.11 para Wifi.

Tabela 11. Tecnologias Recomendadas.

\begin{tabular}{|c|c|}
\hline Estudos & Tecnologias \\
\hline $\begin{array}{c}\text { S1, S2, S3, S5, } \\
\text { S7, S10, S11, } \\
\text { S12, S13, S16, } \\
\text { S17, S18, S19, } \\
\text { S21, S22 }\end{array}$ & Não cita \\
\hline & $\begin{array}{c}\text { Uso de tecnologia de rede padrão, tais como } \\
\text { S4, S6, S8, S14, } \\
\text { S15, S20, S23 }\end{array}$ \\
& $\begin{array}{c}\text { Camada física: Web Portal, OFTP over ISDN/ENX } \\
\text { Tecnologias de GPS, ITS; Para conectividade: 5G, } \\
\text { Wifi, V2X (inclui V2V - Vehicle to Vehicle, V2I - Vehicle } \\
\text { to Infrastructure). }\end{array}$ \\
\hline & $\begin{array}{c}\text { Na camada física WAN, GPRS or DSL, na camada de } \\
\text { comunicação DDS (Data Distribution Service for Real- } \\
\text { time Systems) or JMS (Java Message Service), na }\end{array}$ \\
& $\begin{array}{c}\text { camada de dados Hadoop for batch data processing, } \\
\text { and the integration with the SQL and NoSQL }\end{array}$ \\
& $\begin{array}{c}\text { paradigms. Para as outras camadas não foram citadas } \\
\text { tecnologias. }\end{array}$ \\
\hline
\end{tabular}

Logo, percebe-se que o uso de padrões específicos da área é a estratégia mais 
comum para recomendação de tecnologias nas ARs de SoS. Uma possível explicação para a escassez de recomendações de tecnologias pode ser a natureza abstrata e instanciável de uma AR. ARs possuem a prerrogativa de gerarem uma família de arquiteturas concretas para um domínio. Neste sentido, a recomendação de tecnologias específicas poderia induzir erroneamente a ideia de que sua instanciação estaria atrelada ao uso das tecnologias prescritas. Desta feita, entende-se que, ainda que seja plausível recomendar tecnologias como uma forma de auxiliar os arquitetos na derivação de arquiteturas concretas, os estudos mostraram que os autores preferiram não fazê-lo.

QP 2.6. Como cada AR lida com a interoperabilidade de seus constituintes?

Outro aspecto de interesse da pesquisa realizada foi como cada AR implementa a necessidade de interoperabilidade entre os constituintes do SoS. Esse quesito da interoperabilidade foi apresentado de diversas formas pelas ARs, sendo o mais citado o uso de constituintes que estejam de acordo com normas e padrões já existentes, promovendo assim maior possibilidade de interoperabilidade. Esta orientação aparece nos estudos S4, S5, S7, S8, S9, S11 e S17. Entretanto, o S20 propõe a criação de uma autoridade coletiva que discuta e crie padrões específicos para a AR. O estudo S15 sugere o desenvolvimento dos constituintes de forma coletiva. O S10 propõe uma camada de interoperabilidade em sua estrutura. $\mathrm{O}$ estudo $\mathrm{S} 2$ sugere descartar constituintes que não estejam nos padrões de interoperabilidade. Finalmente, o A6 e o S22 deixam este aspecto de interoperabilidade por conta de uma camada de negócio.

Além dessas formas de interoperabilidade, outro estudos tratam essa questão por meio da criação de políticas que definem quais constituintes poderiam participar do SoS (S1), da realização de uma análise do perfil do constituinte (S12) e do uso de funcionalidades de tradução (S14). A EIRA (European Interoperability Reference Architecture) também foi considerada como uma forma de implementar a interoperabilidade (S23). Os demais estudos não deixam claro como lidar com a interoperabilidade.

Assim como na questão anterior, o tema da interoperabilidade entre os constituintes também está bem atrelado ao uso de padrões para o desenvolvimento do SI constituintes. Alguns estudos deixam por conta de padrões já existentes levando em consideração que eles já tratam das questões de interoperabilidade, mas também é trazida a ideia de se criar padrões para os constituintes que queiram participar do SoS.

Camadas de interoperabilidade e gerenciamento também são indicadas como responsáveis por prover a interoperabilidade servindo de elo entre os constituintes. A camada de tradução foi outro método indicado, sendo ela uma camada capaz de prover a comunicação entre tecnologias diferentes.

QP 2.7. Quais os atributos de qualidade priorizados?

A última questão respondida durante a leitura completa dos estudos se refere aos atributos de qualidade priorizados nas ARs de acordo com o que cada estudo considera importante para o SoS. Segurança foi o atributo que mais se destacou. A Tabela 12 apresenta quais atributos foram priorizados em cada estudo. $\mathrm{Na}$ tabela algumas ARs consideram mais de um atributo de qualidade. Entretanto, os estudos S6, S10, S11, S13, S16, S18, S19 e S21 não consideram atributos de qualidade. 
Tabela 12. Atributos de Qualidade Priorizados.

\begin{tabular}{|c|c|}
\hline $\begin{array}{c}\text { Atributos de Qualidade } \\
\text { (AQ) }\end{array}$ & Estudos \\
\hline Segurança & $\mathrm{S} 1, \mathrm{~S} 3, \mathrm{~S} 4, \mathrm{~S} 5, \mathrm{~S} 14, \mathrm{~S} 15, \mathrm{~S} 17, \mathrm{~S} 20, \mathrm{~S} 23$ \\
\hline Escalabilidade & $\mathrm{S} 3, \mathrm{~S} 9, \mathrm{~S} 14$ \\
\hline Disponibilidade $\mathrm{S} 8, \mathrm{~S} 9$ \\
\hline Confiabilidade & $\mathrm{S} 1, \mathrm{~S} 15, \mathrm{~S} 17$ \\
\hline Performance & $\mathrm{S} 12$ \\
\hline Reúso & $\mathrm{S} 5$ \\
\hline Flexibilidade & $\mathrm{S} 2, \mathrm{~S} 22$ \\
\hline Não tratam de AQ & $\mathrm{S} 6, \mathrm{~S} 10, \mathrm{~S} 11, \mathrm{~S} 13, \mathrm{~S} 16, \mathrm{~S} 18, \mathrm{~S} 19$ e S21 \\
\hline
\end{tabular}

$30,43 \%$ dos estudos analisados apresentam ARs com foco em dois atributos de qualidade (S1, S3, S4, S5, S14, S15, S17). O atributo de segurança foi aquele que recebeu maior destaque, seguido de Disponibilidade (4 estudos), Escalabilidade e Confiabilidade (3 estudos). Tais atributos são considerados importantes quando se trata de SoS. A segurança mostrou-se um atributo particularmente importante devido à coexistência de múltiplos sistemas interoperando e à necessidade de preservar informações sensíveis, apesar do alto grau de troca de informações entre os constituintes.

\subsubsection{QP 3. Qual o principal tipo de contribuição do estudo?}

A Tabela 13 apresenta as principais contribuições apresentadas em cada estudo incluído no mapeamento sistemático. A principal contribuição encontrada nos estudos selecionados foi a proposição de Arquitetura de Referência para SoS em 73,9\% dos artigos. Entretanto, também foram identificados métodos de criação de ARs em 26,1\% dos estudos.

Tabela 13. Contribuição dos Estudos.

\begin{tabular}{|c|c|}
\hline Contribuição & Estudos \\
\hline Arquitetura & $\begin{array}{c}\mathrm{S} 1, \mathrm{~S} 3, \mathrm{~S} 4, \mathrm{~S} 5, \mathrm{~S} 7, \mathrm{~S} 9, \mathrm{~S} 11, \mathrm{~S} 12, \\
\mathrm{~S} 13, \mathrm{~S} 21, \mathrm{~S} 22, \mathrm{~S} 23\end{array}$ \\
\hline Método & $\mathrm{S} 2, \mathrm{~S} 6, \mathrm{~S} 8, \mathrm{~S} 10, \mathrm{~S} 16, \mathrm{~S} 20$ \\
\hline
\end{tabular}

Foram identificados $30 \%$ dos estudos que contribuem com um método para criação de ARs. Entretanto, a maioria dos estudos apresentam ARs para domínios 
específicos. Pode-se concluir que a principal contribuição do trabalho é a própria AR. Há, portanto, espaço para contribuições mais amplas que ajudem no desenvolvimento da AR, como modelos de referência para desenvolvimento de ARs.

\subsubsection{QP 4. Quais as direções futuras para a pesquisa?}

Dentre os estudos incluídos, cinco (S1, S4, S10, S15 e S20, que representam $21,74 \%$ do total) não apresentam quaisquer direções futuras, nem mesmo para suas próprias pesquisas. Muitos dos demais estudos indicam formas de evoluir suas ARs como direções de pesquisa. Dentre eles, notou-se que muitos dos estudos indicam direções de pesquisa semelhantes, mas genéricas, tais como (i) apresentar mais detalhes do método de concepção apresentado (S2), (ii) replicar o estudo aplicando a AR em outros domínios ou cenários atípicos (S3, S6, S7, S11, S16, S19) (iii) aumentar a abrangência da AR (S5), (iv) tratar questões de segurança na $\operatorname{AR}(\mathrm{S} 8, \mathrm{~S} 14)$, (v) acrescentar mecanismos de variabilidade na arquitetura (S9), (vi) realizar auto avaliação (S12), (vii) acrescentar mecanismos para garantir confiabilidade (S13), (vii) conceber versões mais simplificadas da AR (S17), (ix) acrescentar mecanismos de verificação (S18), (x) amadurecer a AR para tratar a questão de interoperabilidade (S21), que ainda não é tratada no contexto da AR relatada e (xi) fazer estudos de caso reais e instaciações (S22, S23). Logo, não há uma identificação explícita de oportunidades de pesquisa, o que leva ao conteúdo da seção seguinte, em que foi realizada uma meta-análise para extrair oportunidades de pesquisa não explicitamente mencionadas, mas passíveis de inferência a partir da análise dos resultados e evidências obtidas.

\section{Discussão}

A partir das respostas às questões de pesquisa, procedeu-se com uma análise sobre as evidências obtidas, isto é, uma análise de todas as respostas de modo a oferecer uma classificação das áreas de interesse em AR para SoS bem como oportunidades de pesquisa, em alinhamento com as prerrogativas de Petersen et al. [Petersen et al., 2015].

As áreas de interesse de AR para SoS foram extraídas pela observância das respostas às questões de pesquisa. Percebeu-se que smart grid, segurança (no contexto militar) e transporte (público ou privado, como veículo próprio) foram os domínios mais frequentes. Quanto às formas de concepção de $\mathrm{AR}$ para $\mathrm{SoS}$, percebeu-se que não há uma convergência. Ainda que o ProSA-RA tenha sido mencionado, notou-se preponderância do uso de modelos de referência e processos como relevantes no apoio à concepção de AR. Verificou-se também a necessidade de aprimorar o rigor científico nas formas de avaliar AR, uma vez que a maioria das avaliações foi realizada por prova de conceito, enquanto que experimentos controlados e simulações foram mais escassos. E, no que tange a atributos de qualidade, percebeu-se foco em segurança.

A partir da visão geral obtida dos resultados, foi possível observar as lacunas (e oportunidades de pesquisa) ainda não exploradas, as quais discutimos a seguir:

ARs para constituintes. Percebeu-se, mediante análise das ARs encontradas nos estudos incluídos, que todas as ARs foram propostas para o SoS como um todo, com exceção do estudo S23, que tratou de uma AR para carros inteligentes e conectados, ou seja, para 
constituintes que farão parte de um sistema de tráfego inteligente em uma cidade inteligente. Esta lacuna é uma notável oportunidade de pesquisa. Ela mostra que ainda há um vasto horizonte a ser explorado no que tange às ARs para guiar a concepção de sistemas que possam vir a fazer parte de um SoS. Tal lacuna estende-se por vários domínios, uma vez que o único domínio explorado foi o automobilístico para fins de transporte privado em ambiente urbano, o que leva ao próximo desafio levantado.

ARs para domínios específicos. Ainda que uma quantidade substancial de domínios tenha sido tratada (tais como Smart grid, segurança e militar), nota-se um número ainda maior de domínios relevantes que não foram cobertos ou que ainda demandam mais atenção, tais como Educação, Saúde, Agronegócio e Financeira/Bancária. O domínio de cidades inteligentes também apresenta um rol de oportunidades. O domínio industrial também tem diversas possibilidades de desenvolvimento de ARs, entre tantos outros domínios possíveis.

ARs para SoIS. A busca realizada incluiu as palavras-chave relativas a SoIS. Entretanto, observou-se que nenhum dos estudos tratava de ARs no contexto de SoIS. Logo, este tipo de evidência mostra-se como uma oportunidade de pesquisa em aberto para ser tratada nos próximos anos, dado que os SoIS podem tornar-se mais presentes nos próximos anos devido à evolução das pesquisas na área [Teixeira et al., 2019]. Sendo ARs específicas de domínio, pode-se tratar de SoIS em diversos domínios, tais como o Educacional, Segurança, Casas Inteligentes, entre outros. Outra perspectiva possível são as ARs para os constituintes destes SoIS.

Uso de métodos mais maduros para avaliação de AR. Observou-se também que, dentre os métodos de avaliação utilizados, a maioria utilizou-se de prova de conceito para avaliação da AR relatada. Logo, nota-se uma necessidade de amadurecimento dos meios de avaliação de AR, seja durante o processo de concepção dela, seja depois do início de sua utilização na academia ou na indústria. Logo, há uma lacuna relativa ao uso de estudos de caso e experimentos controlados para avaliar a AR durante seu uso. O uso de avaliações de especialistas e questionários aplicados aos interessados das áreas de aplicação das ARs também aparece na literatura como métodos confiáveis, embora ainda sejam pouco aplicados.

Uma vez identificadas as oportunidades de pesquisa, pode-se proceder às ameaças à validade do estudo conduzido.

\section{Ameaças à Validade}

Durante o desenvolvimento do mapeamento sistemático foram identificadas algumas ameaças à validade dos resultados apresentados comuns aos estudos sistemáticos [Kitchenham et al. 2009, Felizardo et al. 2017]. Um fator que pode ser considerado uma ameaça é a criação da string de busca, que pode limitar o escopo da pesquisa, excluindo assim alguns estudos válidos para a análise. Para mitigar esta ameaça e maximizar a cobertura da string, a string foi elaborada com base em (i) estudos de referência na área, 
(ii) uso de sinônimos coletados na literatura e a devida avaliação da chave de busca com especialistas em SoS e AR, e (iii) execução de buscas piloto com a string para garantir que ela fosse efetiva. A propósito, a partir das buscas piloto, houve refinamento da string para que ela chegasse ao formato utilizado neste estudo.

A seleção dos estudos também pode ser um fator de viés, uma vez que foi realizada por apenas um pesquisador, sendo que a participação de um dos especialistas só foi requerida em situações de dúvida do pesquisador principal. Ainda existe a possibilidade de falhas no processo de extração dos dados. Para mitigar tais ameaças, a seleção de estudos e extração dos dados foi realizada de forma sistemática, auditável e repetível. Ademais, todas as atividades foram realizadas com supervisão de dois dos pesquisadores envolvidos neste artigo. Todas as dúvidas que surgiram foram dirimidas durante o processo através de discussões com o especialista em SoS com o intuito de minimizar ao máximo qualquer interferência na imparcialidade da análise. Portanto, a confiabilidade da seleção e extração foi devidamente assegurada. Em uma possível replicação do mapeamento, a realização da seleção e extração de dados poderiam ser executadas por, pelo menos, três pesquisadores. Com isso, poderia ser reduzida a possibilidade de falha ou viés no processo.

Nem todas as bases comuns a estudos sistemáticos foram contempladas. Ainda assim, para minimizar os impactos desta decisão, ressalta-se que foram utilizados três motores de busca abrangentes (Scopus, Engineering Village e ACM DL) e duas bases bibliográficas, menos abrangentes, mas complementares aos motores de busca (Science Direct e IEEE Xplore). O uso de cinco bases diferentes e complementares reduz o risco de estudos relevantes não terem sido incluídos e ameniza a ameaça levantada. Entretanto, destaca-se que uma atualização da busca pode ser realizada com bases que não tenham sido cobertas.

Outra ameaça à validade da pesquisa é a relativa pequena quantidade de estudos retornados. Como apenas 23 estudos se enquadram nos parâmetros definidos no protocolo, é possível que a força de evidência estatística dos dados obtidos seja questionável. Entretanto, ressalta-se que os estudos incluídos configuram o estado da arte relativo a ARs para SoS, uma vez que filtros adicionais não foram incluídos e todos os estudos incluídos constituem, de fato, o conjunto de todos os estudos primários retornáveis relativos ao tema utilizando a chave de busca e as bases mencionadas. Ademais, outros estudos de mapeamento existentes na literatura foram publicados em veículos renomados e possuem números de estudos incluídos semelhantes, tais como [Valle et al., 2020] (21 estudos incluídos) e [Gatica et al., 2018] (22 estudos incluídos), mostrando que (i) a quantidade de estudos é relevante, dado o contexto e as condições em que a busca foi realizada e (ii) é comum que o número de estudos seja relativamente reduzido em áreas de investigação que em ascendência, tais como SoIS, em que o mapeamento incluiu 25 estudos [Teixeira et al, 2019] e este mapeamento, uma vez que os estudos começam a aparecer em 2005, reduzindo as ameaças à validade e mostrando que há, em contraste, oportunidades de pesquisa ainda a serem exploradas.

\section{Considerações Finais}

O objetivo deste artigo foi apresentar os resultados de um mapeamento sistemático sobre 
o uso de arquiteturas de referência (ARs) no contexto de SoS. A partir da análise dos trabalhos identificados foi possível notar que a área de SoS ainda é pouco explorada no tocante a ARs. 23 estudos foram incluídos. Os estudos analisados focam, principalmente, em ARs para o SoS de um ponto de vista macro em detrimento de seus constituintes, mediadores ou links de interoperabilidade, para os quais ARs não foram identificadas. Tais arquiteturas foram concebidas com base em diferentes modelos de desenvolvimento. Com este mapeamento sistemático, foi possível conhecer o panorama de estudos publicados sobre ARs em SoS, além de caracterizar os tipos de AR desenvolvidas e como estão sendo avaliadas. Alguns domínios foram cobertos (tais como smart grid e segurança). Por outro lado, também foi possível verificar que ainda há muitas oportunidades de pesquisa, tais como (i) a criação de ARs nos mais diversos domínios, incluindo Educação, Saúde e Finanças, (ii) propostas de AR no contexto de Sistemas de Sistemas de Informação (SoIS), e (iii) ARs para constituintes de SoS.

ARs são arquiteturas abstratas que encapsulam decisões de projeto e recomendações de tal sorte que as arquiteturas concretas derivadas delas exibam qualidade e possam dar origem a sistemas mais robustos, perenes e confiáveis. O domínio de SoS possui ainda muitos desafios a serem explorados. A propósito, os SoIS são considerados um dos Grandes Desafios em Sistemas de Informação para a Década de 2016 a 2026 [Graciano Neto et al., 2017c]. Portanto, explorar ARs no contexto de SoS e SoIS pode não apenas trazer um elevado de grau de qualidade para os sistemas derivados de tais arquiteturas, mas também contribuir para o avanço no estado da arte nas áreas de SoS, SoIS e cidades inteligentes, que são instâncias notáveis dessa classe de sistemas. Espera-se que as oportunidades de pesquisa identificadas neste estudo possam, portanto, fomentar o avanço no desenvolvimento de ARs para o contexto de SoS nos anos vindouros.

\section{Referências}

Assaad, M. A., Talj, R., Charara, A. (2019). Autonomous driving as system of systems: Roadmap for accelerating development, in SoSE. IEEE.

Boscarioli, C., Araujo, R. M., Maciel, R. S. P. (2017) I GranDSI-BR - Grand Research Challenges in Information Systems in Brazil 2016-2026. Special Committee on Information Systems (CE-SI). Brazilian Computer Society (SBC). ISBN: [978- 857669-384-0]. 184p.

Bures, T., Hnetynka, P., Plasil, F. (2014). Strengthening Architectures of Smart CPS by Modeling Them As Runtime Product-lines, in 17th International ACM Sigsoft Symposium on Component-based Software Engineering, pp. 91-96.

Cadavid, H., Andrikopoulos, V., Avgeriou, P. (2020). Architecting systems of systems: A tertiary study, in Information and Software Technology, Volume 118, February 2020 .

Carbon, R., Johann, G., Muthig, D., Naab, M. (2008). A method for collaborative development of systems of systems in the office domain, in 12th International IEEE Enterprise Distributed Object Computing Conference, pp. 339-345. 
Chang, C. F., Tan, P. H., Tam, S. K. (2009). Managing systems of systems interoperability - federated soa and reference architectures, in INCOSE International Symposium, pp. 199-212.

Bondar, S., Hsu, J.C., Pfouga, A., Stjepandić, J. (2017). Agile digital transformation of System-of-Systems architecture models using Zachman framework, Journal of Industrial Information Integration, v. 7: 33-43.

Delsing, J. (2017). Local Cloud Internet of Things Automation: Technology and Business Model Features of Distributed Internet of Things Automation Solutions, in IEEE Industrial Electronics Magazine, 8-21.

Felizardo, K. R., Nakagawa, E. Y., Fabbri, S. C. P. F., Ferrari, F. C. (2017). Revisão sistemática da literatura em Engenharia de Software: teoria e prática. Elsevier, first edition.

Garces, L., Nakagawa, E. Y. (2017). A process to establish, model and validate missions of systems-of-systems in reference architectures, in Symposium on Applied Computing, ACM.

Gatica, D., Ponce, F., Noël, R., Astudillo, H. (2018). Characterizing Architectural Evaluations and Identifying Quality Attributes addressed in Systems-of-Systems: A Systematic Mapping Study, in 37th International Conference of the Chilean Computer Science Society (SCCC).

Graciano Neto, V. V., Cavalcante, E., El Hachem, J., Santos, D. S. (2017a). On the Interplay of Business Process Modeling and Missions in Systems-of-information Systems, in JSOS at ICSE. IEEE.

Graciano Neto, V. V., dos Santos, R. P., Viana, D., Araujo, R. (2017b). Towards a Conceptual Model to Understand Software Ecosystems Emerging from Systems-ofInformation Systems. In Software Ecosystems, Sustainability and Human Values in the Social Web (pp. 1-20). Springer, Cham.

Graciano Neto, V. V., Oquendo, F., Nakagawa, E. Y. (2017c). Smart systems-ofinformation systems: Foundations and an assessment model for research development. In: I GranDSI-BR: Grand Research Challenges in Information Systems in Brazil 20162026. (pp. 13-24). Sociedade Brasileira de Computação.

Graciano Neto, V. V., Horita, F., Cavalcante, E., Rohling, A, El-Hachem, J., Santos, D., Nakagawa, E. Y. (2018). A Study on Goal Specification for Systems-ofInformation Systems: Design Principles and a Conceptual Model, in SBSI. ACM.

Graciano Neto, V. V., Lebtag, B. G. A., Teixeira, P. G., Batista, P. E. P., Lopes, V. C., El-Hachem, J., Buisson, J., Oquendo, F., Fernandes, J., Ferreira, F., Santos, R. P., Viana, D., Cavalcante, E., Kassab. M., Mohsin, A., Oliveira, R., Neves, V., Nakagawa, E. Y. (2021). Expanding Frontiers: Settling an Understanding on Systems-ofInformation Systems. Technical Report Available at: https://arxiv.org/abs/2103.14100.

Guessi, M., Oliveira, L.B.R., Nakagawa, E.Y. (2010). Representation of Reference Architectures: A Systematic Review. In: Proceedings of the 23rd International 
Conference on Software Engineering \& Knowledge Engineering (SEKE'2011), Eden Roc Renaissance, Miami Beach, USA, 1-6.

Guessi, M., Graciano Neto, V. V., Bianchi, T., Felizardo, K. R., Oquendo, F., Nakagawa, E. Y. (2015). A Systematic Literature Review on the Description of Software Architectures for Systems of Systems, in 30th Annual ACM Symposium on Applied Computing, 1433-1440.

Kitchenham, B., Brereton, O. P., Budgen, D., Turner, M., Bailey, J., Linkman, S. (2009). Systematic literature reviews in software engineering-a systematic literature review. Information and software technology, 51(1):7-15.

Lana, C. A., Guessi, M., Antonino, P. O., Rombach, D., Nakagawa, E. Y. (2019). A Systematic Identification of Formal and Semi-Formal Languages and Techniques for Software-Intensive Systems-of-Systems Requirements Modeling, in IEEE Systems Journal, Volume: 13, pp. 2201 - 2212

Lee, H., Hong, S. G., Lee, K. B. (2018). An Internet of Things system architecture for aiding firefighters in the scene of disaster, in Journal of Information Processing Systems.

Lopes, A. J. and Lezama, R., Pineda, R. (2011). Model Based Systems Engineering for Smart Grids as systems of systems, in Procedia Computer Science, pp. 441-450.

Maciel, R. S. P., David, J. M. N., Claro, D., Braga, R. (2017). Full interoperability: Challenges and opportunities for future information systems. Sociedade Brasileira de Computação.

Maier, M. W. (1998). Architecting principles for systems-of-systems. Systems Engineering: The Journal of the International Council on Systems Engineering, 1(4), 267-284.

Mendes, A., Loss, S., Cavalcante, E., Lopes, F., Batista T. (2018). Mandala: an AgentBased Platform to Support Interoperability in Systems-of-systems, in 6th SESoS. ACM.

Morkevicius, A., Bisikirskiene, L., Bleakley, G. (2017). Using a systems of systems modeling approach for developing Industrial Internet of Things applications, in SoSE. IEEE.

Nakagawa, E. Y., Oquendo, F., Becker, M. (2012). RAModel: A Reference Model for Reference Architectures. WICSA/ECSA 2012. pp. 297-301.

Nakagawa, E. Y., Guessi, M., Maldonado, J. C., Feitosa, D., Oquendo, F. (2014). Consolidating a process for the design, representation, and evaluation of reference architectures. In 2014 IEEE/IFIP Conference on Software Architecture (pp. 143-152). IEEE.

Nakagawa, E.Y., Allian, A., Oliveira, B., Sena, B., Paes, C., Lana, C., Feitosa, D., Santos, D., Zaniro, D., Dias, D., Horita, F., Affonso, F. J., Abdalla, G., Vicente, I., Duarte, L., Felizardo, K., Garcés, L., Oliveira, L., Gonçalves, M., Morais, M. G., Guessi, M., Silva, N., Bianchi, T., Volpato, T., Graciano Neto, V. V., Zani, V., Manzano, W. (2017). Software Architecture and Reference Architecture of Software-intensive 
Systems and Systems-of-Systems: Contributions to the State of the Art, USP, São Carlos, SP.

Neureiter, C., Uslar, M., Engel, D., Lastro, G. (2016). A Standards-Based Approach for Domain Specific Modelling of Smart Grid System Architectures, in SoSE. IEEE.

Park, S., Park, Y.B. (2016). ITE Arbitrator: A Reference Architecture Framework for Sustainable IT Ecosystems, in SESoS. IEEE.

Pavlovic, M., Gawrondeutsch, T., Neureiter, C., Diwold, K. (2016). SGAM Business Layer for a Local Flexibility Market, in CIRED Workshop. IEEE.

Percivall, G. S., Alameh, N. S., Caumont, H., Moe, K. L., Evans, J. D. (2013). Improving disaster management using earth observations-GEOSS and CEOS activities, in IEEE Journal of Selected Topics in Applied Earth Observations and Remote Sensing, pp. 1368-1375.

Pérez, J., Díaz, J., Garbajosa, J., Yagüe, A., Gonzalez, E., Lopez-Perea, M. (2015). Towards a Reference Architecture for Large-scale Smart Grids System of Systems, in Third International Workshop on Software Engineering for Systems-of-Systems, pp. 5-11.

Petersen, K., Vakkalanka, S., Kuzniarz, L. (2015). Guidelines for conducting systematic mapping studies in software engineering: An update. Information and Software Technology, 64:1-18.

Rohling, A. J., Graciano Neto, V. V., Ferreira, M. , G. V., dos Santos, W. A., Nakagawa, E. Y. (2019). A reference architecture for satellite control systems. Innovations in Systems and Software Engineering, v. 15, n. 2, pp. 139-153.

Seghiri, A., Belala, F., Teniou, A. A. (2018). Towards software architectural description for systems of systems: Case of a maritime transport SoS, in International Conference on Internet and e-Business. ACM.

Teixeira, P. G., Lopes, V. H. L., Dos Santos, R. P., Kassab, M., Graciano Neto, V. V. (2019, May). The status quo of systems-of-information systems. In 2019 IEEE/ACM 7th International Workshop on Software Engineering for Systems-of-Systems (SESoS) and 13th Workshop on Distributed Software Development, Software Ecosystems and Systems-of-Systems (WDES) (pp. 34-41). IEEE.

Valle, P. H. D., Garcés, L. Guessi, M., Martínez-Fernández, S., Nakagawa, E.Y. (2020). Approaches for Describing Reference Architectures: A Systematic Mapping Study, in XXIII Congresso Ibero-Americano em Engenharia de Software (CIBSE).

Volpato, T., Oliveira, B. R. N., Rodriguez, L. M. G., Capilla, R., Nakagawa, E. Y. (2017). Two perspectives on reference architecture sustainability. ECSA (Companion), pp. 188-194.

Yuan, E., Wenzel, G. (2005). Assured counter-terrorism information sharing using Attribute Based Information Security (ABIS), in IEEE Aerospace Conference, pp. 112 . 
Yuanfang, W, Hongren, Z., Zhongliang, J. (2008). A framework of municipal management information systems (MMIS) in e-government, in IEEE International Technology Management Conference (ICE), pp. 1-8.

Walewski, J. W., Heiles, J. (2016). Using the view model to contextualize and explain system-of-systems architecture models, in 11th System of Systems Engineering Conference (SoSE), pp. 1-6.

Zhu , S. T., Wong , R. W., McDonough , C. A., Roy, R. R., Fine, J. M. e Reiling, J. P. (2009). Army enterprise architecture technical reference model for system interoperability, in IEEE Military Communications Conference, pp. 1-6. 


\section{APÊNDICE 1}

Formulário de Pesquisa.

\begin{tabular}{|c|c|c|}
\hline Questão Formulário & Tipo de Questão & QP \\
\hline Ano da publicação & Questão Aberta & 1 \\
\hline Veículo de publicação & Questão Aberta & 1 \\
\hline $\begin{array}{l}\text { Qual método de modelagem foi utilizado } \\
\text { para criar a AR? }\end{array}$ & Questão Aberta & 2.1 \\
\hline $\begin{array}{l}\text { Qual linguagem foi utilizada para } \\
\text { representar a AR? }\end{array}$ & Questão Aberta & 2.1 \\
\hline Qual o domínio da AR? & Questão Aberta & 2.2 \\
\hline Qual o estilo arquitetural utilizado? & $\begin{array}{l}\text { Questão fechada com as seguintes } \\
\text { opções: } \\
\text {-Camadas, } \\
\text {-Centrada na base de dados, } \\
\text {-Cliente-Servidor, } \\
\text {-Pipes and Filters, } \\
\text {-Outro }\end{array}$ & 2.3 \\
\hline Como a AR foi validada? & Questão Aberta & 2.4 \\
\hline Quais tecnologias essa AR recomenda? & Questão Aberta & 2.5 \\
\hline $\begin{array}{l}\text { Como cada AR lida com a } \\
\text { interoperabilidade de seus constituintes? }\end{array}$ & Questão Aberta & 2.6 \\
\hline $\begin{array}{l}\text { Algum atributo de qualidade foi priorizado } \\
\text { no desenvolvimento da AR? Quais? }\end{array}$ & Questão Aberta & 2.7 \\
\hline Qual a contribuição do artigo? & $\begin{array}{l}\text { Questão fechada com as seguintes } \\
\text { opções: } \\
\text {-Aplicação, } \\
\text {-Arcabouço, } \\
\text {-Arquitetura, } \\
\text {-Ferramenta, }\end{array}$ & 3 \\
\hline
\end{tabular}




\begin{tabular}{|c|c|c|}
\hline & $\begin{array}{l}\text {-Método, } \\
\text {-Métrica, } \\
\text {-Modelo, } \\
\text {-Processo, } \\
\text {-Outro }\end{array}$ & \\
\hline $\begin{array}{l}\text { Como se classifica o artigo pelos critérios } \\
\text { de Petersen et al. (2015)? }\end{array}$ & $\begin{array}{l}\text { Questão fechada com as seguintes } \\
\text { opções: } \\
\text {-Artigo de opinião, } \\
\text {-Artigo filosófico, } \\
\text {-Avaliação, } \\
\text {-Proposta de solução, } \\
\text {-Relato de experiência, } \\
\text {-Validação }\end{array}$ & 4 \\
\hline $\begin{array}{l}\text { Qual o método de pesquisa adotado } \\
\text { pelos critérios de Petersen et al. (2015)? }\end{array}$ & $\begin{array}{l}\text { Questão fechada com as seguintes } \\
\text { opções: } \\
\text {-Estudo de caso, } \\
\text {-Experimento Controlado, } \\
\text {-Pesquisa ação, } \\
\text {-Prototipagem, } \\
\text {-Simulação, } \\
\text {-Survey, } \\
\text {-Outro }\end{array}$ & 5 \\
\hline Quais os problemas em aberto? & Questão Aberta & 6 \\
\hline
\end{tabular}




\section{APÊNDICE 2}

Distribuição dos Estudos por Local de Publicação.

\begin{tabular}{|c|c|}
\hline Veículo de Publicação & Estudos \\
\hline IEEE Aerospace Conference Proceedings & S1 \\
\hline IEEE International Enterprise Distributed Object Computing Conference (EDOC) & S2 \\
\hline IEEE International Technology Management Conference (ICE) & S3 \\
\hline IEEE Military Communications Conference (MILCOM) & S4 \\
\hline $\begin{array}{c}\text { Annual International Symposium of the International Council on Systems } \\
\text { Engineering (INCOSE) }\end{array}$ & S5 \\
\hline Procedia Computer Science & S6 \\
\hline $\begin{array}{c}\text { IEEE Journal of Selected Topics in Applied Earth Observations and Remote } \\
\text { Sensing }\end{array}$ & S7 \\
\hline $\begin{array}{c}\text { International ACM Sigsoft Symposium on Component-based Software } \\
\text { Engineering }\end{array}$ & S8 \\
\hline Conference System of Systems Engineering (SoSE) & $\begin{array}{l}\mathrm{S} 10, \mathrm{~S} 13 \\
\mathrm{~S} 17, \mathrm{~S} 20\end{array}$ \\
\hline CIRED Workshop & S11 \\
\hline $\begin{array}{l}\text { International Workshop on Software Engineering for Systems-of-Systems } \\
\text { (SESoS) }\end{array}$ & S9, S12 \\
\hline IEEE Industrial Electronics Magazine & S14 \\
\hline Journal of Industrial Information Integration & S15 \\
\hline Symposium on Applied Computing & S16 \\
\hline International Conference on Internet and e-Business & S18 \\
\hline Journal of Information Processing & S19 \\
\hline International Conference on Software Engineering Workshops (ICSEW) & S21 \\
\hline
\end{tabular}




\begin{tabular}{|c|c|}
\hline Sensors & S22 \\
\hline Science of Computer Programming & S23 \\
\hline
\end{tabular}

